\title{
Cellular throughput optimization by game-based power adjustment and outband D2D communication
}

\author{
Jinsong Gui* [D and Kai Zhou
}

\begin{abstract}
Device-to-device (D2D) communication is a promising option for the fifth generation (5G) mobile communication network to reduce energy consumption and increase throughput, which makes high throughput applications possible. Also, the improvement for energy and spectrum efficiency is critical in such applications. Without occupation of cellular spectrum resources, outband D2D communication is increasingly applied to high throughput applications to increase spectrum supply. However, energy efficiency is still a key issue that needs to be addressed. Moreover, the overall energy efficiency in cellular networks is severely limited by cell-edge devices. Therefore, in this paper, we apply multi-hop relay-aided outband D2D communication to cellular networks and propose a game-based power adjustment method to address throughput optimization problem. Firstly, we model an interaction relationship of power adjustment for each transmission path as a potential game, where a new utility function is designed for each player (i.e., the receiving end of a transmission path) to evaluate its action gain and to determine whether taking action or not. And then, it is proved that the utility function is an ordinal potential function (OPF) and the game of power adjustment is an ordinal potential game $(\mathrm{OPG})$, which guarantees the convergence of game decision process. Moreover, we propose a new game decision algorithm, which has quicker convergence speed than the existing typical algorithm. In addition, we design a networkassisted distributed processing architecture for solving throughput optimization problem, including receiving mode selection, verification for relay selection, and transmission power adjustment, which can ease the burden of centralized processing. The experimental results show that our scheme is superior to the existing typical work in terms of throughput, delay, energy efficiency, continuous service ability, and convergence performance.
\end{abstract}

Keywords: Cellular throughput optimization, Game-based power adjustment, Multi-hop relay, Outband D2D communication

\section{Introduction}

Internet traffic has witnessed an unprecedented growth because of high throughput applications, including data intensive and content-rich applications (e.g., virtual reality, video streaming, content sharing, and online game). Internet traffic reached $10 \mathrm{~GB}$ per capita in 2016 while it will reach 30 GB per capita by 2021 , where traffic from wireless and mobile devices will account for more than $63 \%$ of total Internet traffic by 2021 [1].

Moreover, a huge amount of small smart objects are expected to connect to Internet due to the wide application of industrial Internet of Things (IoT) [2-7]. Furthermore,

\footnotetext{
* Correspondence: jsgui06@163.com

School of Information Science and Engineering, Central South University, Changsha 410083, China
}

Cisco has predicted that there are 50 billions of small IoT devices by 2020, while Huawei even predicts that the figure will reach 100 billions by 2025 [8]. Such explosive growth for wireless access demand has been pushing academia and industry to explore new cellular architectures and paradigms [9-11] to improve network throughput on the basis of the limited frequency resources.

Deployment of various types of small cells is an agile and energy-efficient scheme to meet throughput demand. However, a large number of deployed base station (BS) will pose challenges for capital expenditure (CAPEX) and operating expenditure (OPEX) to network operators. Device-to-device (D2D) communication is widely regarded as one of key technologies in the fifth generation (5G) mobile communication 
network [12], which does not require operators to invest heavily but can improve network throughput.

Usually, there are two lines of efforts for D2D to improve overall throughput for network system. The one is to offload cellular traffic from BSs if a source device can communicate with a destination device in a direct manner or with the help of D2D relay (referred to as traffic offload), while the other is to enhance access service capability of BSs by using D2D relay function (referred to as capacity and coverage enhancement).

According to the spectrum type used for D2D communication, the available literatures are usually categorized into two major groups (i.e., inband D2D and outband D2D), where inband D2D can be further divided into two communication modes (i.e., underlaying and overlaying). In underlaying mode, the same licensed spectrum can be shared by cellular links and D2D links, and thus, interference will occur not only between a cellular link and a D2D link but also among D2D links. In contrast, dedicated licensed spectrum can be given to D2D links in overlaying mode so that D2D links are not affected by cellular links. However, in overlaying mode, there are still interferences among D2D links.

Different from inband D2D, outband D2D communication uses unlicensed band. In this paper, the motivation for choosing outband mode is that such mode does not occupy the cellular spectrum resources and thus increases the spectrum supply. In addition, based on the standard of wireless interfaces (i.e., IEEE 802.21), various heterogeneous networks (e.g., WiFi, LTE, and WiMAX) can be integrated to bring a smooth and hidden switchover between different network interfaces, which has attracted more and more researchers to explore outband D2D communications.

Compared with works in terms of inband D2D, there are relatively smaller works in terms of outband D2D. Also, the relatively more outband D2D works focus on traffic offload, while relatively smaller outband D2D works address capacity and coverage enhancement.

The most recent works in [13-15] focus on cellular capacity and coverage enhancement by employing outband D2D communication mode. The authors in $[13,14]$ address cellular downward throughput enhancement problem under delay constraint by executing transmission mode selection for each user equipment (UE), while the authors in [15] increase energy constraint for receiving mode selection and extend relaying range. Therefore, the latter outperforms the former in terms of outband D2D link lifetime, as well as cellular downlink throughput.

However, they do not consider any power adjustment on any transmission path. Therefore, there is room for both optimizing energy efficiency and controlling cochannel interference between outband D2D links. Also, they adopt a centralized processing architecture, which will lead to a heavy burden in a large network. We address the above problems in this paper and apply multi-hop relay-aided outband D2D communication to cellular networks and present a game-based power adjustment method to address throughput optimization problem. The main contributions are follows:

1) We model an interaction relationship of power adjustment for each transmission path as a potential game, in which a new utility function is proposed for each player (i.e., the receiving end of a transmission path) to evaluate its action gain and to determine whether taking action or not.

2) We prove that the utility function is an ordinal potential function (OPF) and the game of power adjustment is an ordinal potential game (OPG), which guarantees the convergence of game decision process.

3) We present a new algorithm for game decision process, which has quicker convergence speed than the existing typical algorithm.

4) We design a network-assisted distributed processing architecture for solving throughput optimization problem, including receiving mode selection, verification for relay selection, and transmission power adjustment, which can ease the burden of centralized processing.

The remainder of the article is conceived as follows. Firstly, Section 2 summarizes the related researches on cellular throughput optimization by D2D communications. Then, Section 2 gives the detailed scheme for throughput optimization for cellular networks with multi-hop outband D2D communications. Moreover, Section 3 evaluates the performance of the proposed scheme and discusses the simulation results. Finally, Section 4 summarizes the conclusions of this paper.

\section{Related work}

A comprehensive survey on D2D communication can be found in [16-18]. Especially in inband overlaying and outband modes, D2D links do not interfere with cellular links, but there will still be serious interference between D2D links without efficient resource management and interference control.

In recent researches involving D2D overlaying communications, many works focus on the efficient utilization of the dedicated spectrum resources and the improvement of throughput.

The literature [19] thinks that, although overlay D2D mode can avoid the interference between cellular links and D2D links by dedicating spectrum resources for D2D links, the optimal usage of the dedicated spectrum resources among D2D links is still a concern. Also, how 
to enhance the system throughput and the energy efficiency has been explored through allocating the available resource blocks (RBs) reasonably and adjusting the corresponding transmission powers.

The literature [20] explores spectrum-power trading for D2D overlaying communications. The authors consider the spectrum-power cooperation between D2D UEs and cellular UEs, where D2D UEs help to relay the messages of cellular UEs in exchange for bandwidth from cellular UEs for D2D communications.

The literature [21] presents a framework based on stochastic geometry for enhancing throughput in D2D overlaying cellular networks, where D2D UEs send data with a fixed transmit power, while cellular UEs and D2D relays do so by using the channel inversion power control under maximum transmit power constraint.

The literature [22] proposes an efficient resource allocation algorithm to maximize spatial reuse in D2D overlaying cellular networks and greedily determines the number of the required RBs when giving a set of D2D requests.

The literature [23] studies the transmission capacity for multi-mode D2D communication (including direct overlaying D2D), where the authors focus on the comprehensive analysis of transmission capacity for all different types of D2D communication.

Based on the overlay spectrum sharing approach and green relay BSs, the literature [24] proposes a novel architecture to facilitate D2D communications for Internet of Things (IoT) in heterogeneous cellular networks.

The literature [25] thinks that, in D2D overlaying cellular networks, link scheduling and power control have become the main research topics for enhancing spectrum and energy efficiency. Especially, the scheduling of the D2D links is one of the most essential issues to efficiently exploit the spectrum resources.

In the related works in terms of outband D2D communications, the relatively more literatures pay attention to traffic offload (e.g., the works in [26-33]). The authors in [26] explore the performance of outband D2D communications (i.e., WiFi Direct communications) in urban environments and show that WiFi Direct links generally outperform cellular links in terms of data rate. The authors in [27] also think that it is an attractive solution to offload cellular traffic onto D2D connections in WiFi Direct links. However, they focus on a fast and efficient method research for device/service discovery.

The literature [28] exploits WiFi Direct communications for service delivery enhancement, and the corresponding outband D2D-based solutions can achieve benefits in terms of content delivery time and energy saving. The literature [29] proposes a scheme for integrating outband D2D mode into cellular networks and estimates network performance from offloading cellular load onto outband D2D links. The simulation results show that the scheme can improve network throughput and energy efficiency.

To offload cellular traffic onto links between proximate user devices, the literature [30] proposes a system model, which couples cellular links and outband D2D links. The authors demonstrate that the scheme improves the performance of spatial reuse and reduces the interference impact.

The authors in [31, 32] propose a clustering-based solution. The devices in each constructed cluster can directly communicate with the cluster head in a WiFi Direct link, where the cluster head can directly communicate with the BS in a cellular link. This scheme can reduce the signal interference by employing a transmission path consisting of some short links instead of using a long link, and also lower data traffic that can be sent locally by D2D mode instead of going through the BS. Therefore, the clustering-based scheme can offload a large amount of data traffic from cellular networks.

Similar to the literature [31, 32], the literature [33] also divide cellular devices into clusters, where only the device with the best channel condition can communicate with the BS on behalf of the entire cluster, and the WiFi band is utilized to relay traffic within the cluster. Also, they provide analytical models for such system and study the impact of several payoff distribution methods based on coalitional game theory.

Although the number of literatures is relatively small, there are some outband D2D works for capacity and coverage enhancement. For example, the literature [34] explores how outband D2D communication is used to improve uplink throughput in cellular networks, while the literature [35] establishes outband D2D links by combining multiuser with opportunistic use of WiFi bands. These schemes can improve both data rate for cell-edge devices and overall throughput for systems, but they hardly consider the quality of service (QoS) constraint of outband D2D relay selection.

The authors in $[13,14]$ propose the D2D opportunistic relay with QoS enforcement (DORE) scheme, which can improve cellular downlink throughput by selecting one outband D2D relay for each receiving end and specifying a delay constraint condition for D2D relay selection. The literature [15] presents the method for downlink throughput optimization by multi-hop relay-assisted outband D2D communications (DTO-MROD), which extends the number of outband D2D relays from one to two and thus further improve cellular downlink throughput for whole system (in particular, cell-edge devices).

Although there is no co-channel interference between a cellular link and an outband D2D link, there may be mutual interference between outband D2D links. Power 
adjustment mechanisms [36] can efficiently manage co-channel interference. In this respect, game theory is often used in power control modeling.

The authors in $[37,38]$ adopt a game-theoretic approach to model the distributed power allocation problem as a non-cooperative game and derive an iterative algorithm based on nonlinear fractional programming and Lagrange dual decomposition. The literature [39] considers mutual preferences and satisfactions of UEs and thus proposes a game-based stable matching algorithm, where a D2D pair (or a cellular UE)'s preference over a cellular UE (or a D2D pair) is modeled as a maximum energy-efficient problem and a game-theoretic approach is used to solve it.

However, the literatures [37-39] address the problem formulation from the perspective of resource allocation, while we do it from the perspective of throughput optimization. Moreover, they use the ordinary non-cooperative game theory rather than the special potential game theory. Potential games belong to a special set of games, which have a set of potential functions to track the variations in utilities caused by the unilateral deviation of game players. If a potential game has a compact action space, it possesses at least one Nash Equilibria (NE) in pure strategies [40]. Therefore, the potential game theory is very helpful to guide us in game modeling.

The literature [41] formulates power allocation as a potential game, where the goal of each game player is to maximize the sum of the signal-to-interference-plus-noise ratio (SINR) on its used sub-channels. The literature [42] employs potential game theory to study the game convergence for the purpose of minimizing the interference power among game players. The literature [43] models the power allocation problem as a potential game and obtains a local maximum of the objective function by finding a fully distributed solution.

However, the aforementioned literatures on power allocation game are only for inband underlaying or overlaying D2D communications. There is still a lack of power adjustment in the above outband D2D works on capacity and coverage enhancement.

In addition, for any cellular link in cellular networks aided by outband D2D communications, if its transmission power ensures that its receiving end can reach a desired bit error rate (BER) level, a good user experience and satisfaction can be guaranteed. Therefore, excessive transmission power will lead to energy waste, which makes cellular links' power adjustment necessary.

In this paper, we mainly focus on proposals that employ outband D2D communication to improve cellular throughput, where the concept of cellular throughput refers specifically to the throughput transferred through any BS, and the goal of cellular throughput optimization is to minimize the sum of transmission powers on the premise of satisfying the users' application experience. It is noted that only cellular devices with at least two types of wireless interfaces (e.g., LTE and WiFi) can exploit outband D2D. The current multi-radio terminals are already capable of establishing concurrent cellular links and outband D2D links. Therefore, the outband D2D research for cellular throughput enhancement is attracting researchers' attention.

\section{The scheme for throughput optimization by game-based power adjustment and outband D2D communication}

\subsection{System model}

We consider a single cellular network, where all spectrum resources are divided into $N$ equal parts (e.g., $R B s$ ) and scheduled by an $e N B$ (evolved node B). There are $M$ wireless terminals (e.g., user equipment, UE) randomly distributed in this cellular network.

When an UE requests for a transmission service, the $e N B$ allocates a $R B$ to it on the basis of a preset strategy. The allocated $R B$ is used by this UE for receiving downlink data traffic from the $e N B$. When $M$ is greater than $N$, up to $N$ UEs will get a channel (i.e., a $R B$ ) separately. For example, $\mathrm{UE}_{0} \sim \mathrm{UE}_{9}$ shown in Fig. 1 get $\mathrm{C}_{0} \sim \mathrm{C}_{9}$, respectively.

When these $N$ UEs receive downlink data from the $e N B$ simultaneously, the whole downlink throughput depends on the maximum SINR that these $N$ receiving UEs can get. In Fig. 1a, the SINR values of cell-edge receiving $\mathrm{UEs}$ (e.g., $\mathrm{UE}_{1}, \mathrm{UE}_{5}$, and $\mathrm{UE}_{9}$ ) are relatively low since they are far away from the $e N B$, which is not conducive to improving downlink throughput.

Selecting an idle UE (i.e., neither requests for data transmitting service nor acts as a relay) to serve as a relay can enhance a cell-edge receiving UE's SINR and thus improve downlink throughput. Figure $1 \mathrm{~b}$ illustrates that the idle UEs (e.g., $\mathrm{UE}_{a} \sim \mathrm{UE}_{d}$ ) can use WiFi channels (i.e., outband D2D channels) to relay data for the cell-edge receiving $\mathrm{UEs}$ (e.g., $\mathrm{UE}_{1}, \mathrm{UE}_{5}$, and $\mathrm{UE}_{9}$ ) for the purpose of improving downlink throughput. In the next subsection, we will discuss throughput optimization problem for the scenario in Fig. 1.

\subsection{Problem formulation}

We propose an optimizing model to improve downlink throughput for the scenario in Fig. 1 by performing receiving mode selection and determining the number of relays under both delay constraint and remaining energy constraint. We follow the same assumption as that in [13-15], that is, location information of UEs are available at a proximity-based services (ProSe) server according to the design scheme proposed in 3GPP [44-46], and UEs report the channel state information (CSI) of their outband D2D links as well as its delay and 


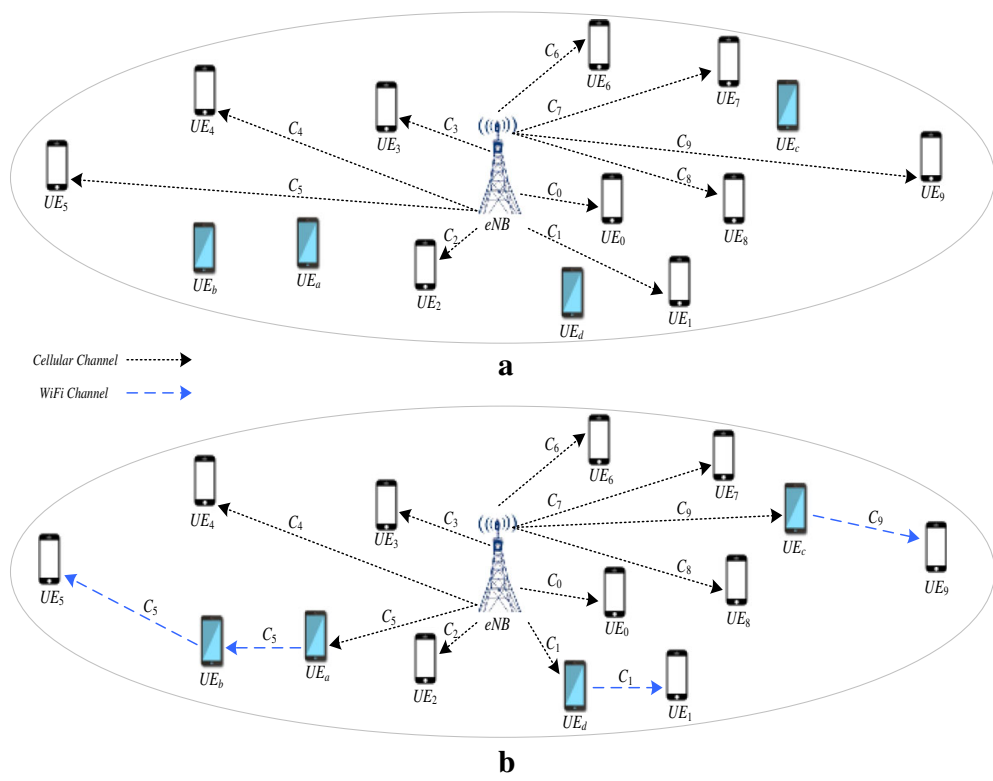

Fig. 1 Example for heterogeneous cellular networks. a The case without the usage of relays $\mathbf{b}$ The case with the usage of relays

remaining energy to the $e N B$. On the basis of Shannon capacity formula, the throughput of UE $i$ who receives its data directly from the $e N B$ can be expressed as follows.

$$
T_{c}^{i}=b_{c}^{i} \cdot \log _{2}\left(1+\gamma_{c}^{i}\right)
$$

In (1), $T_{c}^{i}$ and $\gamma_{c}^{i}$ are the throughput and the SINR for UE $i$ respectively when the $e N B$ sends data to it over a cellular channel, and $b_{c}^{i}$ is the allocated frequency band for UE $i$. When UE $j$ receives data for UE $i$ over cellular channel $b_{c}^{i}$, the corresponding throughput is denoted as $T^{j i}{ }_{c}$ and computed by the following formula.

$$
T_{c}^{j i}=b_{c}^{i} \cdot \log _{2}\left(1+\gamma_{c}^{j}\right)
$$

In (2), $\gamma{ }_{c}^{j}$ is the SINR for UE $j$ when the $e N B$ sends data to it over a cellular channel $b_{c}^{j}$. UE $j$ forwards UE $i$ data over a WiFi channel from UE $j$ to UE $i$, the corresponding throughput is denoted as $T^{j i}{ }_{w}$ and computed by the following formula.

$$
T_{w}^{j i}=b_{w}^{j i} \cdot \log _{2}\left(1+\gamma_{w}^{j i}\right)
$$

In (3), $\gamma{ }^{j i}{ }_{w}$ denotes the UE $i$ ' SINR when UE $j$ sends data to UE $i$ over a WiFi channel, and $b^{j i}{ }_{w}$ is an available WiFi frequency band from UE $j$ to UE $i$. Based on (2) and (3), the throughput from UE $j$ to UE $i$ is estimated by the following formula.

$$
T_{d}^{j i}=\min \left(T_{c}^{j i}, T_{w}^{j i}\right)
$$

In (4), $T^{j i}{ }_{d}$ is the throughput of UE $i$ that receives data from the $e N B$ via UE $j$. When the $e N B$ sends data to UE $i$ via UE $k$ and UE $j$ in turn, the corresponding throughput is denoted as $T_{d}^{k j i}$ and computed as follows.

$$
T_{d}^{k j i}=\min \left(T_{c}^{k i}, T_{w}^{k j}, T_{w}^{j i}\right)
$$

In (5), the $e N B$ sends data to UE $i$ by adopting the transmitting power $p_{O i}$ over a cellular channel, UE $i$ 's SINR is estimated as follows.

$$
\gamma_{c}^{0 i}=\frac{g_{0 i} \cdot p_{0 i}}{N_{i}+F_{i, c}}
$$

In (6), $N_{i}$ is the noise power received by UE $i ; F_{i, c}$ is the interfering power received by UE $i$ over a cellular channel, where $F_{i, c}$ is negligible if co-channel interference between adjacent cells is very small due to the adoption of effective cellular interference control methods; $g_{O i}$ is the attenuation coefficient in the channel from the $e N B$ to UE $i$, and it is subject to effects of shadowing fade, multipath fade, and path loss etc., which is usually estimated and quantified by a receiving end and then feeds back to the corresponding sending end.

When UE $j$ forwards data to UE $i$ by adopting the transmitting power $p_{j i}$ over a WiFi channel, UE $i$ 's SINR is computed as follows.

$$
\gamma_{w}^{j i}=\frac{g_{j i} \cdot p_{j i}}{N_{i}+F_{i, w}}
$$

In (7), $g_{j i}$ is the attenuation coefficient in the channel from UE $j$ to UE $i$, which involves the same factors as that of $g_{O i} ; F_{i, w}$ is the interfering power received by UE $i$ over a WiFi channel, which comes from its surrounding 
transmitting signal in the overlapping spectrum. Therefore, it is computed as follows.

$$
F_{i, w}=\sum_{k \in I_{i}} g_{k i} \cdot p_{k}
$$

In (8), $g_{k i}$ is the attenuation coefficient in the channel from the interfering UE $k$ to the interfered UE $i$, which involves the same factors as that of $g_{O i} ; p_{k}$ is the interfering UE $k$ 's transmission power; $I_{i, \text { nei }}$ is the set of UE $i$ 's interfering sources.

Let $d^{j i}$ denote the link delay from the $e N B$ to UE $i$, where $j$ is a relay and there is no relay if $j=0$. Similarly, let $d^{k i}$ denote the path delay from the eNB to UE $i$, where $j$ and $k$ are the relays in the same path and there is not any relay if $j=0$ and $k=0$.

Based on the above definitions, we can give the following downlink throughput optimization model with energy constraint $e_{t h}$ and delay constraint $d_{t h}$ for $N$ UEs, where the cellular downlink throughput is optimized by selecting a set of D2D relaying links for improving transmission quality.

$$
\begin{aligned}
& \operatorname{Max} \sum_{i \in \Re \backslash\{0\}} \sum_{j \in \mathscr{R} \backslash\{i\}}\left(\left(1-\alpha_{j i}\right) \cdot T_{c}^{i}+\alpha_{j i}\left(\left(1-\alpha_{k j i}\right)\right.\right. \\
& \left.\left.\qquad \cdot T_{d}^{j i}+\alpha_{k j i} \cdot T_{d}^{k j i}\right)\right) \\
& \text { s.t. } \alpha_{j i} \in\{0,1\}, \forall i, j \in\{0,1, \ldots, N\} \\
& \alpha_{i i}=0, i \neq 0 \\
& \alpha_{00}=1 \\
& \sum_{i \in \Re} \alpha_{j i}=1 \\
& \sum_{j \in \Re} \alpha_{j i}-\left(N-1+2 \delta_{0 i}\right) \alpha_{0 i} \leq 0 \\
& \alpha_{k j i} \in\{0,1\}, \forall i, j, k \in\{0,1, \ldots, N\} \\
& \left(\sum_{k \in\{1, \ldots, N\} /\{j, i\}} \alpha_{k j i}\right) \in\{0,1\}, i f \alpha_{j i}=1 \\
& e^{j} \geq e_{t h} ; e^{k} \geq e_{t h} \\
& d^{j i} \leq d_{t h} ; d^{k i i} \leq d_{t h}
\end{aligned}
$$

In (9), $\alpha_{j i}$ and $\alpha_{k j i}$ are the variables for binary decision, where $\alpha_{j i}=1$ means that UE $i$ receives data from UE $j$, while $\alpha_{k j i}=1$ means that UE $k$ forwards UE $i$ 's data from the $e N B$ to $\mathrm{UE} j ; \delta_{i j}$ is set as 1 when $i=j$; otherwise, it is 0 . The $e N B$ is marked as UE 0 only for the convenience of the concept description.

For the first constraint, the decision variable is limited to binary, and the same is true for the sixth constraint. Any UE is forbidden to send data to itself under the second constraint, and the same is true for the third constraint. However, there is the exception of the $e N B$, where it is mainly for notational convenience and there is hardly any physical meaning.
Each UE is forbidden to receive data from the $e N B$ and any relay simultaneously under the fourth constraint, while the number of receiving UEs of a relay is not more than $N-1$ under the fifth constraint.

Based on the seventh constraint, at most one relay is allowed for any UE that also acts as a relay. The eighth constraint specifies that the remaining energy of any relay is not lower than a given energy threshold, while the ninth constraint specifies that the total delay is not more than a given delay threshold.

\subsection{The solving approaches of the proposed optimization model}

For the optimization model proposed in Subsection 3.2 , the idea of centralized algorithms discussed in literature [13-15] can be used for reference. However, the centralized mode is not scalable with the increase of network scale. On the other hand, the control overhead of full distributed mode in a large-scale network environment is unbearable. Therefore, the hybrid mode (i.e., network-assisted distributed mode) is a good compromise. In addition, as mentioned above, the literatures [13, 14] limit outband D2D relays in each transmission path to one outband D2D relay at most is selected for a transmission path in $[13,14]$ and do not consider energy constraint for relay candidates.

Although the literature [15] relaxes the hop-constraint for outband D2D relays and also considers the energy constraint, the work in [15] together with that in $[13,14]$ do not consider power adjustment. The literature [47] considers a distributed power adjustment for the similar downlink throughput model to that proposed in Subsection 3.2, but it aims at effectively controlling co-channel interference in underlay inband D2D mode. However, in this paper, we mainly focus on co-channel interference control between outband D2D relays by using power adjustment. For the sake of reference, we call the scheme in this paper as the throughput optimization method based on power adjustment and outband D2D communication (TOM-PAOD).

Since a user's application experience is particularly vulnerable to BER, it is necessary to identify a BER threshold (e.g., $\left.B E_{t h}\right)$. A user's application experience is met when an actual BER is lower than $B E_{t h}$. The corresponding $\operatorname{SINR}$ (e.g., $y_{t h}$ ) and throughput (e.g., $\left.T_{t h}{ }\right)$ with $B E_{t h}$ is estimated by the following formulas:

$$
\begin{aligned}
& \gamma_{t h}=-2 \ln B E_{t h} \\
& T_{\text {th }}^{i}=b_{\text {cell }}^{i} \cdot \log _{2}\left(1+\gamma_{t h}\right)
\end{aligned}
$$

In the scenario of Fig. 1, for a cellular channel between the $e N B$ and any UE $i$, the $e N B$ 's transmitting power 
should not be lower than $p_{i}^{t h}$, which is computed as follows:

$$
p_{i}^{t h}=\frac{\gamma_{t h} \cdot N_{i}}{g_{i}}
$$

When the eNB's maximum transmitting power is lower than $p_{i}^{\text {th }}$, the UE $i$ should select an outband D2D relay to aid it to receive data from the $e N B$, which may avoid the desired transmission power of the $e N B$ above its maximum transmission power.

From the formulas (10) (12), we know that, the lower BER threshold will require the higher transmission power to ensure the user experience. Also, the user will get higher throughput. However, with the further increase of transmission power, the increase of throughput gets smaller and smaller when compared with the transmitting power. Therefore, very low BER threshold will result in poor energy efficiency.

In practice, different network applications have different BER threshold values. Therefore, when a network application has a large BER threshold, we should not use excessive high transmission power in pursuit of absolute increase in throughput. This is because it is a waste of energy, and also, it may not improve the user experience any more.

According to the goal of cellular throughput optimization in this paper, a BER threshold at a receiving end is an important benchmark for cellular throughput optimization. That is, when each receiving end just meets its own BER threshold, the corresponding throughput value is what we expect. Otherwise, excessive high throughput will not significantly improve the user experience but consume too much energy. In addition, the gap between our scheme and the optimal one depends on the number of receiving UEs that cannot satisfy their BER threshold requirements.

For an outband relaying link $j \rightarrow i$, UE $i$ 's BER value will be not more than $B E_{t h}$ if UE $j$ 's transmitting power is not less than $p^{t h}$ i, which is expressed as the following formula:

$$
p_{j i}^{t h}=\frac{\gamma_{t h} \cdot\left(N_{i}+F_{i}\right)}{g_{j i}}
$$

However, different from any cellular channel in the scenario of Fig. 1, there is the same frequency interference among outband D2D links since the number of non-overlapping frequency bands used by WiFi links is only 3. Also, since the transmission powers of the outband D2D links may be changed at any time, the value of the same frequency interfering value (i.e., $F_{i}$ ) only comes from what is currently known. The current $F_{i}$ may also be adapted due to the available information updated by potential interference sources. The transmitting end (i.e., UE $j$ ) may also be the other receiving ends' interfering source, so its power regulation operation may also lead to a new power regulation operation on the transmitting ends of other WiFi links with overlapping spectrum.

The above interaction process will continue until all interference sources do not change their transmitting powers. Game theory is usually used in such interaction process, since it can offer an efficient distributed framework for various network information systems [48, 49]. Especially, OPG theory can easily characterize the set of NEs, which is convenient for the model design of power regulation process with interactive influence. Therefore, it is suitable for modeling this distributed power adjustment problem [41-43, 50, 51], which involves the design of game decision algorithm besides utility function modeling.

The best response algorithm and better response algorithm are the two kinds of typical game decision algorithms for solving OPG's NE. In the best response algorithm, a game player adopts a value for its transmitting power (i.e., an action) that maximizes its earnings when it has a chance to adjust its transmitting power (i.e., make decision). In the better response algorithm, a game player makes a small decrement in its transmitting power if the power decrement improves its earnings; otherwise, the player still keeps its previous transmitting power.

Although the best response algorithm has a faster convergence speed than the better response algorithm, it has a relatively bad performance in terms of efficiency and fairness. Therefore, the literature [47] proposes the improved response algorithm, which has the similar idea to that of the better response algorithm but improves convergence.

In this paper, we design the similar game decision algorithm to that in [47]. However, the game decision algorithm in [47] is mainly used in transmission power adjustment of potential inband links, while that of this paper is employed for transmission power adjustment of transmission paths from the $e N B$ to each receiving UE. Furthermore, since there are differences between game player types, and utility function modeling methods, the implementation details of the two kinds of game decision algorithms are obviously different. For the convenience of referring to this game decision algorithm, we call it as the improved-a.

In the improved-a, the game players are the transmission paths from the $e N B$ to each receiving UE (i.e., $N$ players), where each path may include one cellular link and one (or two) WiFi link(s), or only 
have one cellular link. Whether a path contains one WiFi link or two WiFi links, the improved-a only performs one power adjustment for a certain transmitting end on the path in each iteration of the game. In order to further accelerate the convergence rate, we improve the improved-a as follows.

When a transmission path contains two WiFi links that all have higher potential throughput than the cellular link in the same path, in each iteration of the game, we first adopt $50 \%$ of the maximum transmission power as a step size of power adjustment of the WiFi link with higher potential throughput and then employ $25 \%$ of the maximum transmission power as a step size of power adjustment of the other WiFi link if the power adjustment of the previous WiFi link is feasible. To distinguish it from the improved-a, we call it as the improved-b.

In both the improved-a and the improved-b, each game player has a utility function that estimates its own benefits and a set of strategies (or actions). For any transmission path, its receiving end (e.g., UE $i$ ) participates in the game on behalf of it by adopting the following utility function.

$$
\mu_{i}(P)=w_{g} \cdot \frac{T}{P}+w_{l} \cdot \frac{T_{i}}{P_{i}}
$$

In (14), the utility can be interpreted as energy efficiency, which is the data rate under unit energy consumption. The utility involves the expected earnings (i.e., $w_{g} T / P$ ) and the actual earnings (i.e., $w_{l} \cdot T_{i} / P_{i}$ ), where the former depends on the potential capacity improvement in the whole network, while the latter only depends on individual effort. $w_{g}$ and $w_{l}$ are the weight value, where $w_{g}+w_{l}=1$.

$T_{i}$ denotes the throughput of the transmission path where the $e N B$ is a sending source, and the UE $i$ is a receiving end. If the UE $i$ does not use any outband D2D relay, $T_{i}$ is estimated by the Formula (1). Otherwise, $T_{i}$ is estimated by the Formula (4) if one outband D2D relay (e.g., UE $j$ ) is adopted by the UE $i$, while $T_{i}$ is estimated by the Formula (5) if two outband D2D relays (e.g., UE $k$ and $\mathrm{UE} j$ ) are selected by the UE $i$.

$P_{i}$ is the sum of transmitting powers of the transmission path where the $e N B$ is a sending source and the UE $i$ is a receiving end. $P_{i}$ only depends on the $e N B$ 's transmission power if the UE $i$ does not use any relay. Otherwise, $P_{i}$ is the sum of the $e N B$ 's transmission power and one relay's transmission power if one relay is adopted, while $P_{i}$ is the sum of the $e N B$ 's transmission power and two relays' transmission powers if two relays are selected. It is worth noting that in order to justify the Formula (14), the denominator cannot be 0 ; and thus, each transmitting end should take a transmitting power that is not less than its specific circuit power.

$T$ denotes the total potential throughput of all the transmission paths from the $e N B$ to each receiving UE in the whole network, while $P$ denotes the sum of transmitting powers of these transmission paths, which is computed as follows:

$$
\left\{\begin{array}{l}
T=\sum_{i \in U=\{1,2, \ldots N\}} T_{i} \\
P=\sum_{i \in U=\{1,2, \ldots N\}} P_{i}
\end{array}\right.
$$

\subsection{The theoretical analysis for potential game}

The convergence of utility function proposed in Subsection 3.3 will be proved in this subsection. To this end, we firstly introduce potential game. According to [52], $\mathrm{Z}=\langle U, A, \mu\rangle$ is usually used to formalize a potential game, in which $U=\{1,2, \ldots, n\}$ denotes a set of game players; the space of all action vectors is denoted as $A=\prod_{i=1}^{n} A_{i}$, in which $A_{i}$ represents the set of the $i$ th game player's actions; $\mu=\left(\mu_{1}, \mu_{2}, \ldots, \mu_{i}, \ldots, \mu_{n}\right)$ is an utility function vector and $\mu_{i}$ is the utility for the $i$ th game player, which measures the preferences over action profiles for all game players.

The $a_{i}$ is a component in vector $a$, where $a_{i} \in A_{i}$ and $a \in A$. Usually, $a=\left(a_{i}, a_{-i}\right)$ represents an action profile, in which $a_{i}$ denotes the player $i$ 's action, and $a_{-i}$ denotes the actions of the other $n-1$ players. Similarly, the set of action profiles for all players, except for the player $i$, is denoted as $A_{-i}=\prod_{j \neq i} A_{j}$. For the NE, OPG, OPF, their formal definitions are given as follows [52].

Definition 1. If $\forall i \in U$ and $\forall a_{i} \in A_{\dot{v}}$ the action profile $a^{*}=\left(a_{i}^{*}, a_{-i}^{*}\right)$ is a NE

$$
\mu\left(a^{*}\right) \geq \mu_{i}\left(a_{i}, a_{-i}^{*}\right)
$$

Definition 2. If there exists a function $F: A \rightarrow \mathbb{R}$ such that $\forall i \in U$ and $\forall a_{-i} \in A_{-i}$ and for all $a_{i} \in A_{i}$ and $b_{i} \in A_{i}$, the $A$ game $\mathrm{Z}=\langle U, A, \mu>$ is an $O P G$

$$
\begin{aligned}
F\left(a_{i}, a_{-i}\right)-F\left(b_{i}, a_{-i}\right) & >0 \Leftrightarrow \mu_{i}\left(a_{i}, a_{-i}\right)-\mu_{i}\left(b_{i}, a_{-i}\right) \\
& >0
\end{aligned}
$$

According to [52], there is an NE if an OPG (e.g., $\mathrm{Z}=<U$, $A, \mu>$ ) can optimize its corresponding OPF (e.g., $F$ ). Therefore, a subset of NEs in a potential game consists of a set of potential maximizers. If a set of potential functions of a potential game can be found, some NEs of the game can found by solving for the potential maximizers.

Theorem 1. The game (i.e., $\mathrm{Z}=\langle U, A, \mu\rangle$ ) is an OPG, in which individual utilities are given by (14). An OPF is given as follows. 


$$
F(P)=\sum_{i \in U=\{1,2, \ldots N\}}\left(w_{g} \cdot \frac{T}{P}+w_{l} \cdot \frac{T_{i}}{p_{i}}\right)
$$

Proof. By applying the asserted OPF in (18), we firstly have $\Delta \mu_{i}=\mu_{i}\left(P_{i}, P \backslash P_{i}\right)-\mu_{i}\left(P_{i}^{i}, P \backslash P_{i}^{i}\right)=$

$$
\begin{aligned}
& w_{g} \cdot\left(\frac{T_{i}+\sum_{j \in U i} T_{j}}{P_{i}+\sum_{j \in U \backslash i} P_{j}}-\frac{T_{i}^{c}+\sum_{j \in U \backslash i} T_{j}}{P_{i}^{c}+\sum_{j \in U \backslash i} P_{j}}\right) \\
& \quad+w_{l} \cdot\left(\frac{T_{i}}{P_{i}}-\frac{T_{i}^{\prime}}{P_{i}^{\iota}}\right)
\end{aligned}
$$

Let $A=\sum_{j \in U \wedge i} T_{j}$ and $B=\sum_{j \in U \lambda i} P_{j}$ : therefore, we have $\Delta$ $\mu_{i}=w_{g} \cdot\left(\frac{T_{i}+A}{P_{i}+B}-\frac{T_{i}^{i}+A}{P_{i}^{i}+B}\right)+w_{l} \cdot\left(\frac{T_{i}}{P_{i}}-\frac{T_{i}^{i}}{P_{i}^{i}}\right)$.

For simplicity and without loss of generality, we only take a transmission path (that only contains one cellular link) for an example, where $T_{i}=b_{c}^{i} \cdot \log _{2}\left(1+\frac{g_{0 i} \cdot p_{0 i}}{N_{i}+F_{i, c}}\right)$ and $T_{i}^{i}=b_{c}^{i} \cdot \log _{2}\left(1+\frac{g_{0 i} \cdot p_{0 i}^{i}}{N_{i}+F_{i, c}}\right)$ according the Formulas (1) and (6). Also, for such path that only contains one cellular link, $P_{i}=p_{0 i}$ and $P_{i}^{i}=p_{0 i}^{i}$.

Similarly $\Delta F=F\left(P_{i}, P \backslash P_{i}\right)-F\left(P_{i}^{i}, P \backslash P_{i}^{i}\right)=$

$$
\begin{gathered}
\left(w_{g} \cdot \frac{T_{i}+\sum_{j \in U \backslash i} T_{j}}{P_{i}+\sum_{j \in U \backslash i} P_{j}}+w_{l} \cdot \frac{T_{i}}{P_{i}}\right)+\sum_{k \in U \backslash i} \\
\left(w_{g} \cdot \frac{T}{P}+w_{l} \cdot \frac{T_{k}}{P_{k}}\right)-\left(w_{g} \cdot \frac{T_{i}^{c}+\sum_{j \in U \backslash i} T_{j}}{P_{i}^{c}+\sum_{j \in U \backslash i} P_{j}}+w_{l} \cdot \frac{T_{i}}{P_{i}^{c}}\right) \\
-\sum_{k \in U \backslash i}\left(w_{g} \cdot \frac{T}{P}+w_{l} \cdot \frac{T_{k}}{P_{k}}\right)=\left(w_{g} \cdot \frac{T_{i}+\sum_{j \in U \backslash i} T_{j}}{P_{i}+\sum_{j \in U \backslash i} P_{j}}\right. \\
\left.+w_{l} \cdot \frac{T_{i}}{p_{i}}\right)-\left(w_{g} \cdot \frac{T_{i}^{c}+\sum_{j \in U \backslash i} T_{j}}{P_{i}^{c}+\sum_{j \in U \backslash i} P_{j}}+w_{l} \cdot \frac{T_{i}^{c}}{P_{i}^{c}}\right)=w_{g} . \\
\left(\frac{T_{i}+A}{P_{i}+B}-\frac{T_{i}^{s}+A}{P_{i}^{c}+B}\right)+w_{l} \cdot\left(\frac{T_{i}}{P_{i}}-\frac{T_{i}^{c}}{P_{i}^{c}}\right)
\end{gathered}
$$

Based on the above, $\Delta F$ has the same sign as that of $\Delta \mu_{i}$. Therefore, according to Definition 2, it is proved that $F(P)$ is an OPF and $\mathrm{Z}=\langle U, A, \mu\rangle$ is an OPG.

\subsection{The hybrid algorithm for energy-efficient downlink throughput optimization}

As mentioned above, we propose a hybrid algorithm for solving the downlink throughput maximization problem described in Subsection 3.2. At the same time, it is our main goal to improve the energy efficiency of the solved results. The proposed hybrid algorithm involves the three main stages: Receiving Mode Selection (RMS), Verification for Relay Selection (VRS), and Transmission Power Adjustment (TPA).
In RMS stage, under the unified coordination of the $e N B$, each receiving UE independently determines whether it receives data directly from the $e N B$, or selects one (or two) relay(s) to forward the data to itself from the $e N B$.

In VRS stage, the $e N B$ adopts a centralized approach to verify whether the selected relays can improve throughput for each receiving UE. In TPA stage, with the aid of the $e N B$, and based on the potential game scheme proposed in Subsection 3.3 and proved in Subsection 3.4, each receiving UE independently adjusts the powers of the transmitting ends on its data receiving path.

With the aid of the $e N B$, any receiving UE $i$ independently run Algorithm 1 to determine its data receiving mode. When UE $i$ receives starting package for receiving mode selection from the $e N B$ (see line 1), it will perform the operations from line 2 to line 25. Also, it may receive the corresponding instructions from the $e N B$ to cancel the selected relays, where the corresponding variables for recording relay channel labels (e.g., $c_{k j}$ and $c_{j i}$ ) as well as variables for recording relay channel occupancy status (e.g., $\alpha_{k j i}$ and $\alpha_{j i}$ ) will be updated or cleared (see lines 26-27).

UE $i$ will determine to receive data directly from the $e N B$, if the BER value of its receiving end is below the threshold $B E_{t h}$. Otherwise, it will select the first relay (see lines 3 13) or further select the second relay (see lines 14 21) if necessary.

As mentioned in the literature [15], since the actual co-channel interference among WiFi links is uncertain, the accurate throughput of such outband D2D links is unavailable. Therefore, a relay should be preselected according to the difference value (e.g., $\Delta T^{j i}$ ) between the link throughput from the $e N B$ to the relaying UE (e.g., $i$ ) and the link throughput from the $e N B$ to the receiving UE (e.g., $j$ ). In this paper, we also adopt such idea (see line 4 and line 15), where $V_{i}$ (or $V_{j}$ ) is the set of all the UEs within the approximate coverage of UE $i$ (or UE $j$ ). According to Formulas (11) (13) in the literature [15], such approximate coverage can be estimated.

In the first relay selection, under the premise for meeting both energy constraint and delay constraint, a receiving UE $i$ will select a relaying UE $j$ with the maximum value for $\Delta T^{j i}$ (see lines $5 \sim 12$ ), and also the allocated WiFi channel's sequence number in the WiFi link from UE $j$ to UE $i$ is stored in $c_{j i}$, where $c_{j i}$ is set as 0 when no WiFi channel is allocated to this WiFi link.

In the second relay selection, a receiving UE $i$ only considers a relaying UE $k$ with the maximum value for $\Delta T^{k j i}$ (see lines 16 20). If this relaying UE $k$ does not meet either energy constraint or delay constraint, this receiving UE $i$ does not continue to choose any other 
relay. Otherwise, if UE $i$ successfully choose the second relay, there are the two consecutive WiFi links on the path from the $e N B$ to UE $i$.

In $802.11 \mathrm{a} / \mathrm{b} / \mathrm{g}$, WiFi frequency band is usually split into 13 basic sub-channels and marked as a set of numeric IDs, ranging from 1 to 13 , where the some sub-channel frequency bands (e.g., sub-channel 1, 6, and 11) do not overlap when the difference between the sequence numbers of their labels is not less than 5. Therefore, in order to ensure any relay receives and sends data simultaneously by using WiFi sub-channels, the non-overlapping WiFi sub-channels should be assigned to these two consecutive WiFi links (see line 18).

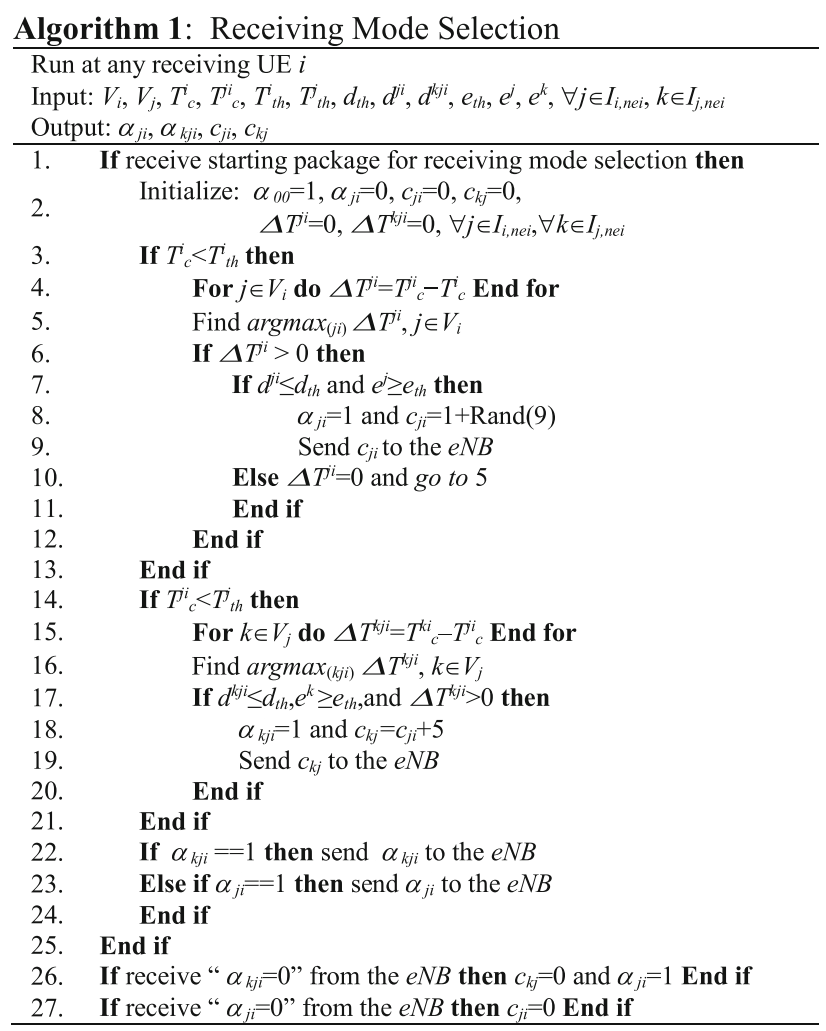

Similar to the RMS stage mentioned in the above, with the aid of the $e N B$, any receiving UE $i$ independently run Algorithms $2 \sim 3$ to adjust the powers of transmitting ends on the data receiving path from the $e N B$ to it. The basic idea for TPA is that, the search space in the set of transmission power levels (i.e., the set of actions in game) is decreased by using large step size search in Algorithm 2, while a sequential small step size search is adopted for solving a suitable transmitting power in Algorithm 3.

Since these two algorithms are run on each receiving UE, there is a need for information exchange (e.g., sending channel state information to the $e N B$, while getting $T$ and $P$ from it) with the $e N B$. In addition, the following data structures need to be defined for all the other algorithms in this paper. (1) $P_{M, M}$ is the relation matrix for WiFi link transmitting power allocation between UEs, where a value of a member (e.g., $p_{j i}$ ) denotes the transmitting power that is assigned to a link from UE $j$ (i.e., $j \in\{1, \ldots, M\}$ ) to UE $i$ (i.e., $i \in\{1, \ldots, M\}$ ). (2) $C_{M, M}$ is the relation matrix for WiFi channel allocation between UEs. If a value of a member (e.g., $c_{j i}$ ) in $C_{M, M}$ is $k$ (i.e., $k \in\{1, \ldots, 13\})$, it means that the WiFi channel $k$ is assigned to a link from UE $j$ (i.e., $j \in\{1, \ldots, M\}$ ) to UE $i$ (i.e., $i \in\{1, \ldots, M\})$. Otherwise, it means that no channel is assigned to the link $j \rightarrow i$.

Once receiving the message packets (including $P_{M, M}$, $C_{M, M}, T, P$ ) from the $e N B$, Algorithm 2 and 3 start. Also, they stop the running process after receiving end package for power adjustment from the $e N B$. Therefore, the WiFi links' transmitting powers will be adjusted under the unified coordination of the $e N B$.

In Algorithm 2, the goal of lines 2 26 is to adapt the powers of transmitters in a data receiving path with the two WiFi links, where the WiFi link with greater throughput capacity will get the power adjustment opportunity if its potential throughput is higher than a given threshold (e.g., $T_{t h}^{k}$ ), and the other WiFi link will also have the power adjustment opportunity if both its potential throughput is higher than $T^{k}{ }_{t h}$ and the power adjustment of the previous WiFi link is feasible.

When the transmitter in the WiFi link with greater throughput capacity transmits at $50 \%$ of the maximum transmitting power, if the throughput of the corresponding receiving path is larger, it employs the new power; otherwise, it uses the original (i.e., maximum) power and reports it to the $e N B$ (see lines $8 \sim 9$ and lines 18 19). If the operation of the WiFi link with greater throughput capacity is successful, the operation of the other WiFi link is performed, where the corresponding transmitting end employs $75 \%$ of the maximum transmitting power, and if the throughput of the corresponding receiving path is larger, it adopts the new value and sends it the $e N B$; otherwise, it employs the maximum transmitting power and reports it to the $e N B$ (see lines 10 14 and lines 20 24).

The goal of lines 27 36 in Algorithm 2 is to control the transmitting powers in any data receiving path with the one WiFi link, where the WiFi link will get the power adjustment opportunity if its potential throughput is higher than $T^{k}{ }_{t h}$. Also, when each transmitting end transmits at $50 \%$ of the maximum transmitting power, if the throughput of the corresponding receiving path is larger, it employs the new power; otherwise, it uses the maximum transmitting power and reports it to the $e N B$ (see lines 32 33). 
Algorithm 2: Coarse Adjustment for Relaying UEs' Transmission Powers

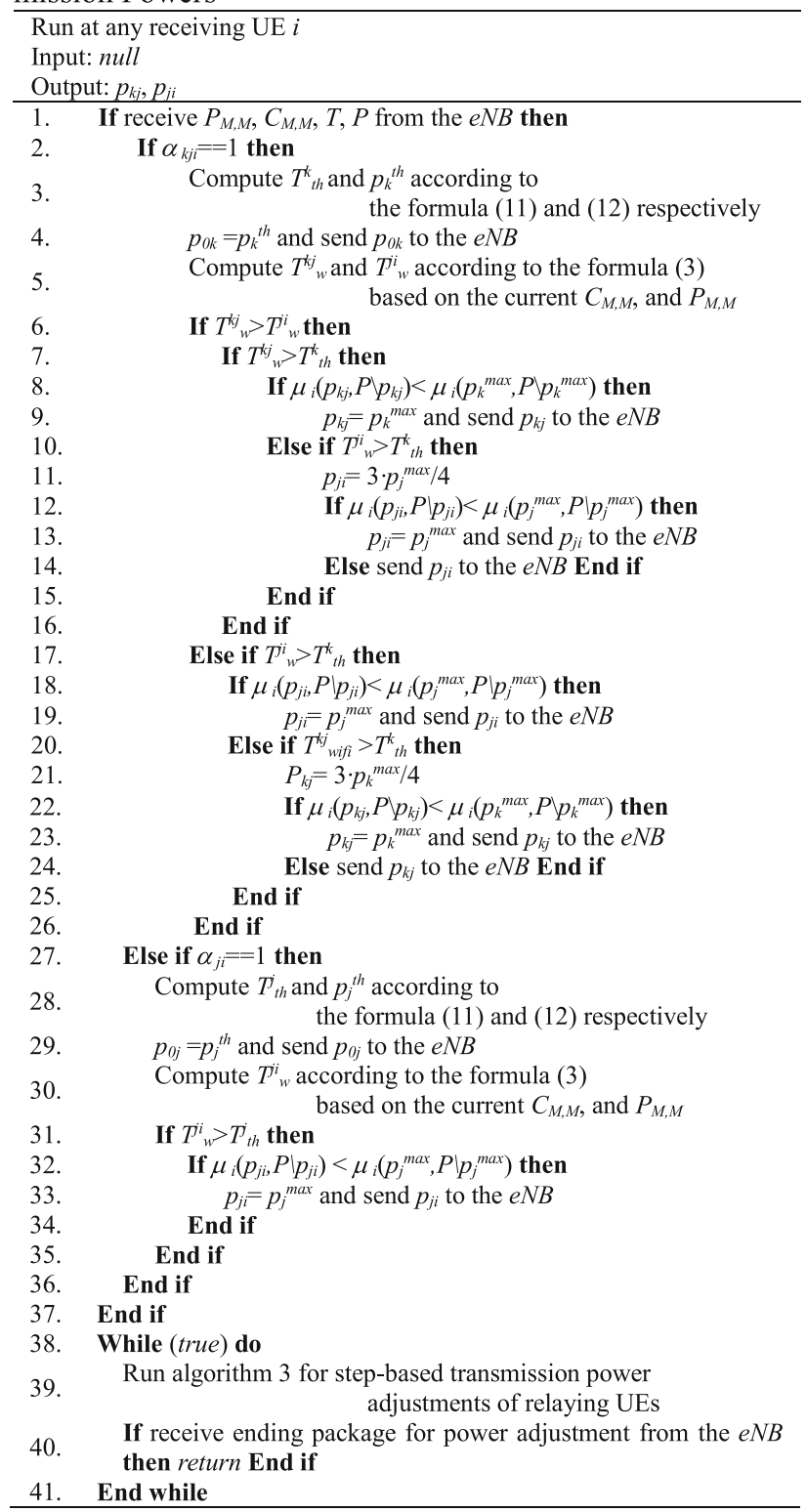

After the above operations end, a receiving UE $i$ will execute Algorithm 3 to find out a suitable transmitting power in a descending order manner until it gets ending package for power adjustment from the eNB (see line 40). The updated package (including $P_{M, M}, C_{M, M}, T, P$ ) will trigger next iteration (see line 1 of Algorithm 3).

In Algorithm 3, the goal of line $2 \sim 10$ is to control the transmitting powers in any data receiving path with the two WiFi links, where the WiFi link with greater throughput capacity will get the power adjustment opportunity if its potential throughput is higher than a given threshold (see lines 5 and 8), and each transmitter reduces a small step size in its current transmitting power and reports the new power value to the $e N B$ if its utility is improved by the new power value (see lines 6 and 9).

The goal of lines 11 16 in Algorithm 3 is to control the transmitting powers in any data receiving path with the one WiFi link, where the WiFi link will get the power adjustment opportunity if its potential throughput is higher than a given threshold (see line 13), and then, each transmitter lowers a small step size in its current transmitting power and reports the new power value to the $e N B$ if its utility is improved by the new power value (see line 14).

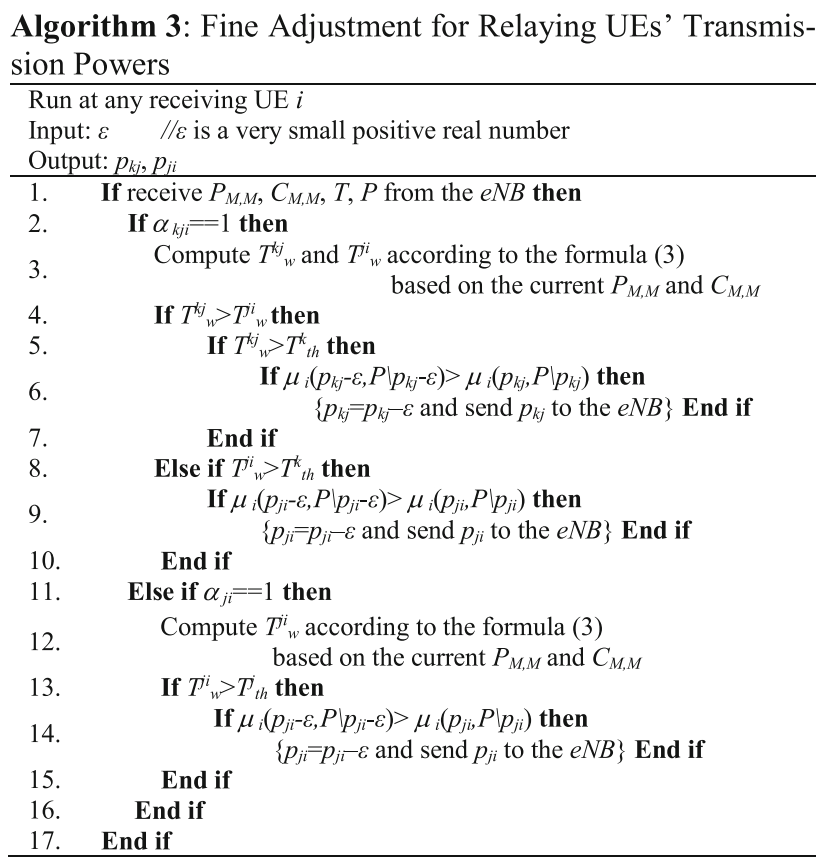

The $e N B$ runs Algorithm 4 to assist each receiving UE to complete receiving mode selection and transmission power adjustment. Firstly, the initialized transmitting powers in all the WiFi links are $50 \%$ of their maximum transmitting powers (see line 2), and the corresponding WiFi channel allocation relation is initialized as unallocated state (see line 3). Then, the eNB schedules all receiving UEs to execute Algorithm 1 by broadcast starting package for receiving mode selection. In a given time, by receiving feedback from each receiving UE, the $e N B$ updates the corresponding values in $C_{M, M}, D, E$ respectively (see lines $5 \sim 10$ ), where $D$ (and $E$ ) are initialized as empty (see line 1) to prepare for keeping identifications on behalf of 2-hop (and 3-hop) date receiving paths.

After verifying whether the preselected outband D2D relaying UEs improve throughput or not (see lines $11 \sim 12)$, the $e N B$ computes $T$ and $P$ according to the Formula (15) and then broadcasts $P_{M, M}, C_{M, M}, T, P$ to all 
the receiving UEs (see lines 13 14) to start the power adjustment process.

Also, the $e N B$ waits for the feedback information for power adjustment in an infinite loop (see lines 15 29), where a label variable (e.g., tag) is firstly set as false (see line 16). In a preset time interval, if there is any changed transmitting power, the label variable $t a g$ is updated as true, and also, $P_{M, M}$ is updated (see lines 17 21).

If the value of tag is equal to true, the $e N B$ recomputes $T$ and $P$ according to the Formula (15) and then continues to initiate power adjustment process by rebroadcasting $P_{M, M}, C_{M, M}, T, P$ to all the receiving UEs (see lines 23 24). Otherwise, it indicates to all the receiving UEs that the power adjustment process ends and then exits from the infinite loop (see line 26 27).

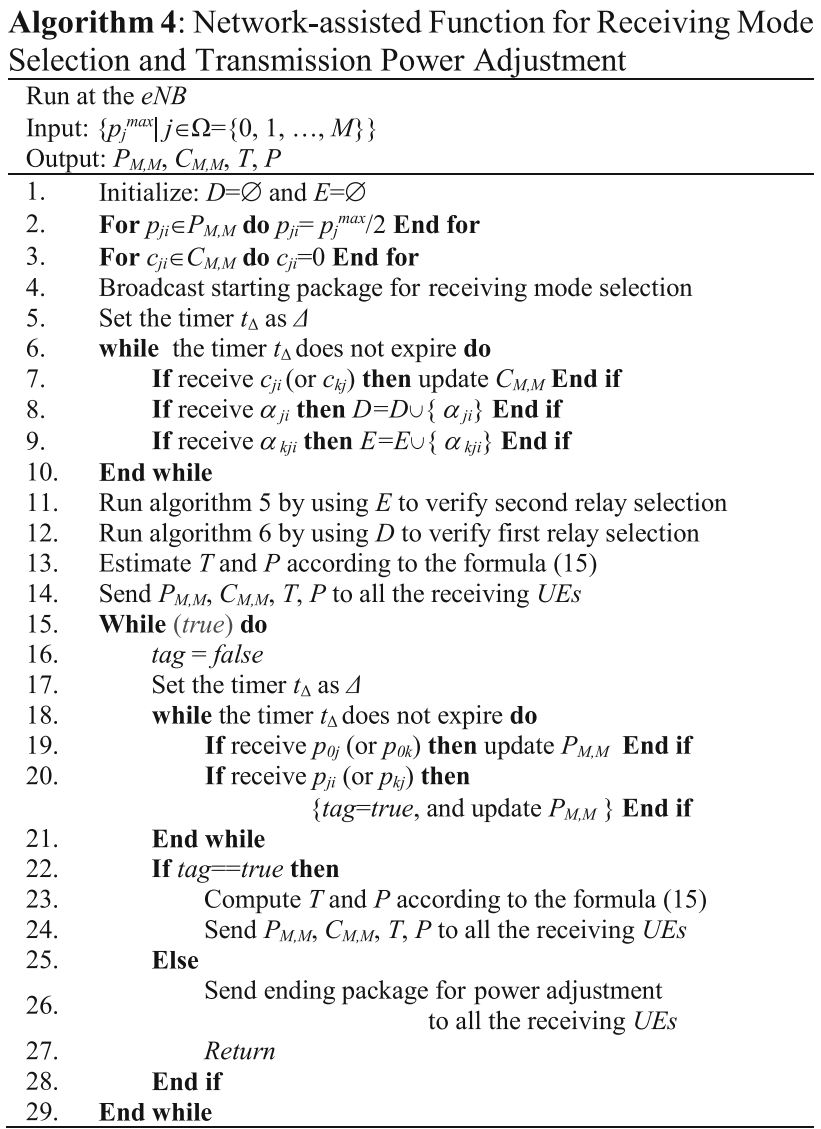

Algorithms 5 and 6 are invoked by the $e N B$ to verify whether the selected potential outband D2D relaying UEs improve throughput or not after each receiving UE determines its data receiving mode. In these two algorithms, we mainly consider the interference sources of a receiving UE (e.g., UE $i$ ) as the transmitting ends of overlapping channel WiFi links in UE $i$ 's adjacent area and denote the set of transmitting ends as its neighboring interference set (i.e., $I_{i, \text { nei }}$ ).

According to the values in $E$, the $e N B$ can check whether all the second preselected relays improve throughput or not by running Algorithm 5. In Algorithm 5, for any 3-hop data receiving path (e.g., $e N B \rightarrow k \rightarrow j \rightarrow i)$, the co-channel interference value at $\mathrm{UE} i$ is estimated in lines $3 \sim 7$, while that at UE $j$ is estimated in lines $8 \sim 12$. Based on the estimated co-channel interference values, the throughput values of the two data receiving paths (i.e., 2-hop path $e N B \rightarrow j \rightarrow i$ and 3-hop path $e N B \rightarrow k \rightarrow j \rightarrow i$ ) are computed respectively (see lines 13 14). If the 2-hop path outperforms the 3-hop path in terms of throughput, the second preselected relay (i.e., UE $k$ ) will be canceled (see lines 15 19). Otherwise, no action will be done.

The Abs (see lines 5 and 10 in Algorithm 5) means the function that solves the absolute value of any pair of arguments, and also, the polynomial value in the bracket denotes overlap ratio for WiFi frequency band. Since the overlap ratio of any pair of consecutive WiFi sub-channels is approximatively $80 \%$ of frequency band in one WiFi sub-channel, this value decreases by $20 \%$ when the difference between their labels increases by 1 .

According to the values in $D$, the $e N B$ can check whether all the first preselected relays improve throughput or not by executing Algorithm 6, where the corresponding description is similar to that in Algorithm 5 and thus omitted in this paper for the sake of space saving.

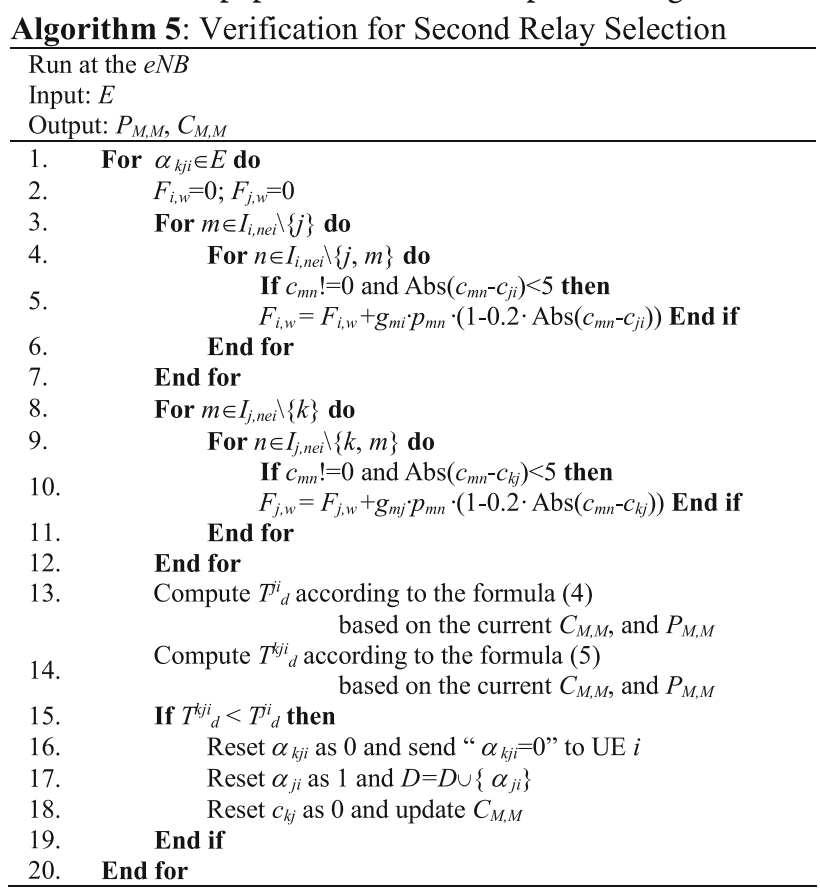




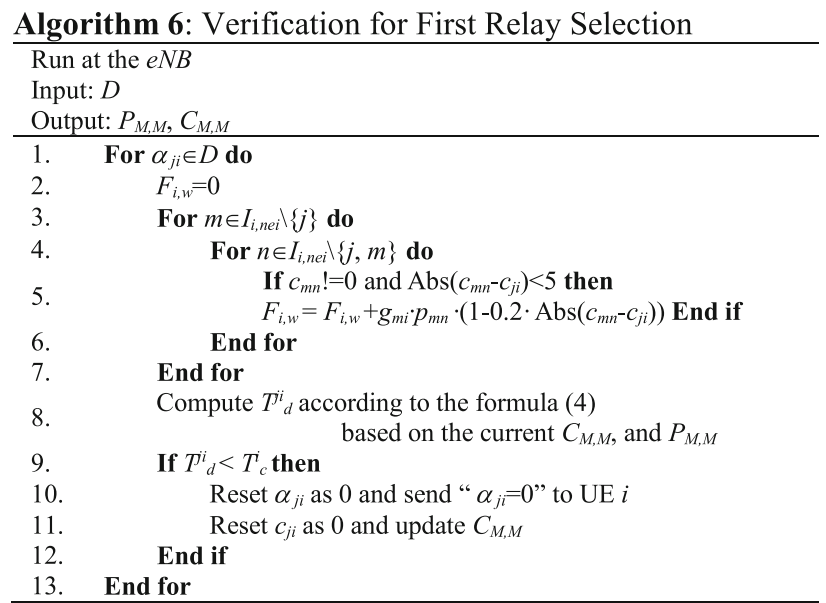

\subsection{TOM-PAOD procedures ProSe-compliant framework}

In a centralized D2D relay/mode selection [44], the service framework consists of registration, collection, decision, activation, communication, and termination, which is also detailed in [15]. Different from the centralized cases, where the process for D2D relay/mode selection is performed by a ProSe Application Server, the corresponding process in Fig. 2 is run by each receiving UE in a distributed manner with the help of network infrastructure.
Therefore, the framework in Fig. 2 adds a step "discovery" in this paper, besides all the steps in the framework in [15]. In the step "discovery," any D2D UE knows its neighbors' IDs by broadcasting a hello message and getting the corresponding response message and then gets the neighbors' relevant information from the ProSe Application Server by sending its neighbors' IDs to this server. In the step "decision," with the help of network infrastructure that executes Algorithms 4 6, each receiving UE executes Algorithms 1 3 and reports the decision result to the ProSe Function which then forwards this result to the ProSe Application Server.

\subsection{Theoretical analysis for the proposed scheme}

We analyze the communication and computational complexity of the TOM-PAOD scheme in this subsection. The total number of UEs in the network is represented as $|\Omega|=M$, and the number of members in any set $V_{i}$ is represented as $\left|V_{i}\right|$. The parameter $x$ is the number of rounds in the power adjustment game. Theorems 2 3 describe the computational complexity of the TOM-PAOD scheme.

Theorem 2. The computational complexity of a receiving $U E$ (e.g., i) is $O\left(\max \left\{\left|V_{i}\right|, x, \max \left\{\left|V_{j}\right| \mid j \in V_{i}\right\}\right)\right.$ in the TOM-PAOD scheme.

Proof. For a receiving UE (e.g., i), the computational complexity of selecting the first relay (see lines 4 to 12 in Algorithm 1) is $O\left(\left|V_{i}\right|\right)$, while the second relay

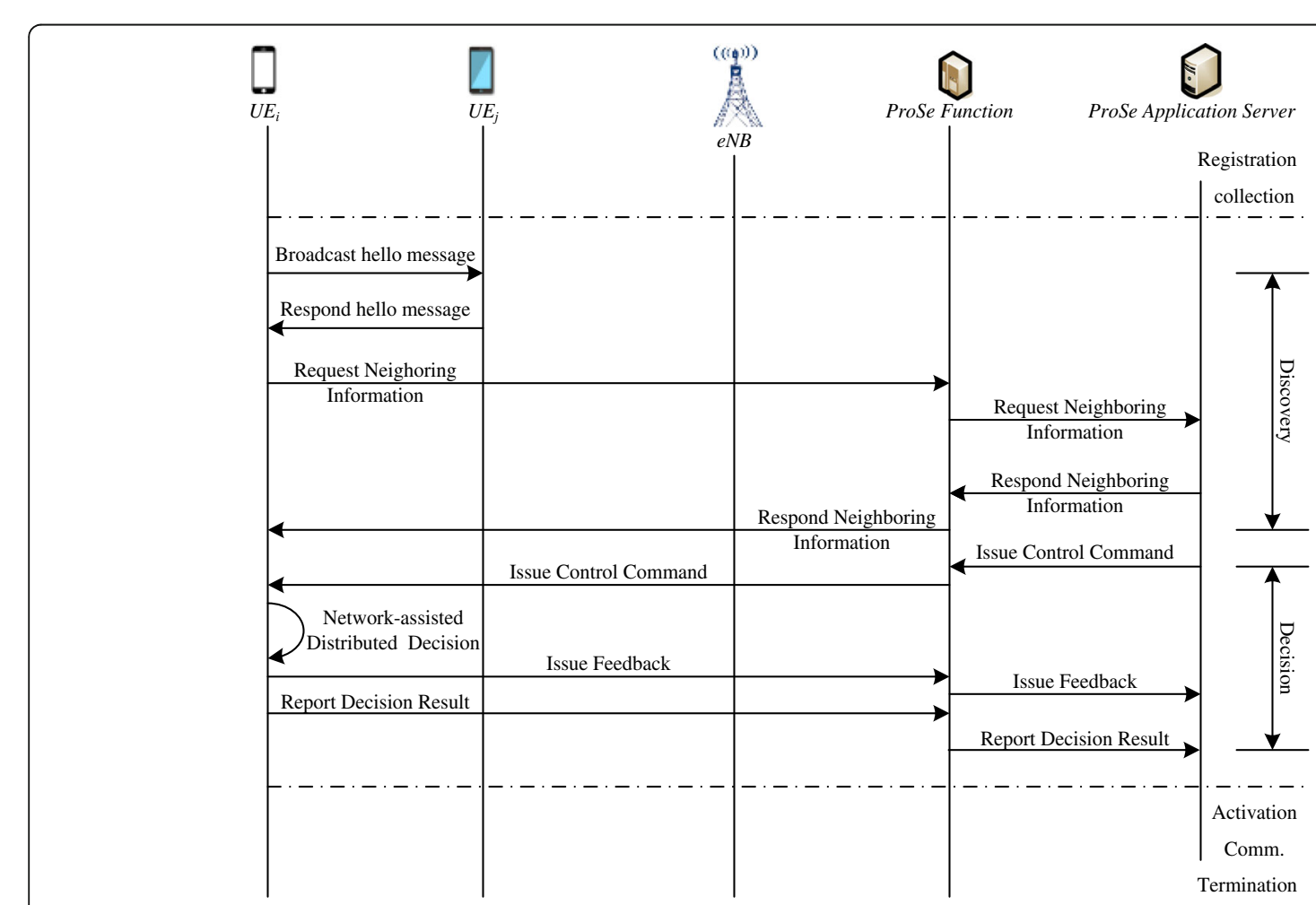

Fig. 2 Schematic protocol overview of TOM-PAOD 
Table 1 The complexity comparison between TOM-PAOD and DTO-MROD

\begin{tabular}{lll}
\hline The compared item & Computational overhead & Communication overhead \\
\hline 1) Receiving UE in TOM-PAOD & $O\left(\max \left\{\left|V_{i}\right|, x, \max \left\{\left|V_{j}\right| \mid j \in V_{i}\right\}\right)\right.$ & $O(x)$ \\
2) Infrastructure node in TOM-PAOD & $O\left(\max \{|D|,|E|\} \cdot \max \left\{||_{i, n e i}|\cdot| V_{j} \mid\right\}\right)$ & $O(\max \{M, x \cdot N\})$ \\
3) Infrastructure node in DTO-MROD & $O\left(N \cdot \max \left\{\left|V_{i, n e i}\right| \cdot\left|V_{j}\right|\right\}\right)$ & $O(M)$ \\
\hline
\end{tabular}

selection (see lines 15 to 20 in Algorithm 1) takes time $O\left(\max \left\{\left|V_{j}\right| \mid j \in V_{i}\right\}\right)$. In the coarse power adjustment (i.e., Algorithm 2), the computational complexity of the receiving UE is $O(1)$, while it is $O(x)$ in the fine power adjustment (i.e., Algorithm 3) since the power adjustment game process are iterated $x$ times. Hence, the resulting computational complexity of a receiving UE (e.g., $i$ ) is $O\left(\left\{\max \left\{\left|V_{i}\right|, x, \max \left\{\left|V_{j}\right| \mid j \in V_{i}\right\}\right\}\right)\right.$.

Theorem 3. In the TOM-PAOD scheme, the computational complexity of a node on the infrastructure is $O\left(\max \{|D|,|E|\} \cdot \max \left\{\left|I_{i, \text { nei }}\right| \cdot\left|V_{j}\right| \mid \quad i \in \Omega, \quad j \in I_{i, \text { nei }}\right\}\right), \quad$ where $I_{i, \text { nei }} \subset V_{i} \subset \Omega=\{1, \ldots, M\}, V_{j} \subset \Omega=\{1, \ldots, M\}$.

Proof. For a node on the infrastructure (e.g., the ProSe Application Server), its computational overhead is mainly spent on verifying second relay selection (see line 11 in Algorithm 4) and first relay selection (see line 12 in Algorithm 4), and estimating $T$ and $P$ according to the Formula (15) (see lines 13 and 23 in Algorithm 4).

Firstly, when running Algorithm 5 to verify second relay selection, the computational complexity is $O\left(|E| \cdot \max \left\{\left|I_{i, \text { nei }}\right| \cdot\left|V_{j}\right| \mid i \in \Omega, j \in I_{i, \text { nei }}\right\}\right)$, while the computational complexity is $O\left(|D| \cdot \max \left\{\left|I_{i, \text { nei }}\right| \cdot\left|V_{j}\right| \mid i \in \Omega, j \in I_{i, \text { nei }}\right\}\right)$ when running Algorithm 6 to verify first relay selection.

Then, when the power adjustment game process are iterated $x$ times, the computational complexity is $O(x)$. Since $O(x)$ is better than $O\left(|E| \cdot \max \left\{\left|I_{i, \text { nei }}\right| \cdot\left|V_{j}\right| \mid i \in \Omega, j \in I_{i,-}\right.\right.$ nei $\})$ or $O\left(|D| \cdot \max \left\{\left|I_{i, \text { nei }}\right| \cdot\left|V_{j}\right| \mid i \in \Omega, j \in I_{i, \text { nei }}\right\}\right)$, the resulting computational complexity is $O\left(\max \{|D|,|E|\} \cdot \max \left\{\mid I_{i,-}\right.\right.$ nei $\left.\left.|\cdot| V_{j}|| i \in \Omega, j \in I_{i, \text { nei }}\right\}\right)$.

Theorems 4 5 describe the communication complexity of the TOM-PAOD scheme.
Theorem 4. The communication complexity of a receiving UE (e.g., i) is $O(x)$ in the TOM-PAOD scheme.

Proof. For a receiving UE (e.g., $i$ ), firstly, it should send its information about location, CSI, and battery level in an information reporting package to ProSe Function, and then, the package is sent to ProSe Application Server. Therefore, the communication complexity of each UE is $O(1)$ when it sends its information packet. Also, it may send request packet and then receive response packet from the $e N B$, where the communication complexity is $O(1)$.

Then, it may receive the starting package for receiving mode selection from the $e N B$ (see line 1 in Algorithm 1) and then return selection results, where the communication complexity is $O(1)$. Finally, it may receive the starting package for power adjustment from the $e N B$ (see line 1 in Algorithm 2) and then return power adjustment results, where the communication complexity is $O(1)$.

After this, it may receive the subsequent package for power adjustment from the $e N B$ (see line 1 in Algorithm 3) and then return power adjustment results, where the operation are iterated $x$ times, and thus, the corresponding communication complexity is $O(x)$. Therefore, the resulting communication complexity of any receiving UE $i$ is $O(x)$ in the TOM-PAOD scheme.

Theorem 5. In the TOM-PAOD scheme, the communication complexity of a node on the infrastructure is $O(\max \{M, x \cdot N\})$.

Proof. For a node on the infrastructure (e.g., the ProSe Application Server), firstly, it must receive $M$ information reporting packages from all UEs as shown in registration and collection stage of Fig. 2, where its

Table 2 The comparison of the characteristics of the three works

\begin{tabular}{|c|c|c|c|}
\hline Characteristic description & Work in [15] & Work in [47] & Work in this paper \\
\hline 1) Solution deployment architecture & Centralized & Hybrid & Hybrid \\
\hline 2) Spectrum used for D2D links & $\begin{array}{l}\text { Unlicensed } \\
\text { band }\end{array}$ & Licensed band & Unlicensed band \\
\hline 3) Receiving mode selection decision & Centralized & Distributed & Distributed \\
\hline 4) Verification for relay selection & Need & No need & Need \\
\hline 5) Transmission power adjustment & No & Yes & Yes \\
\hline $\begin{array}{l}\text { 6) Calculated object of game utility } \\
\text { function }\end{array}$ & No & Link & Path \\
\hline 7) The game player type & No & Link's receiving end & Path's receiving end \\
\hline $\begin{array}{l}\text { 8) Convergence rate of game decision } \\
\text { algorithm }\end{array}$ & No & $\begin{array}{l}\text { Better than the better response algorithm } \\
\text { in [52] }\end{array}$ & $\begin{array}{l}\text { Better than the game decision algorithm } \\
\text { in [47] }\end{array}$ \\
\hline
\end{tabular}




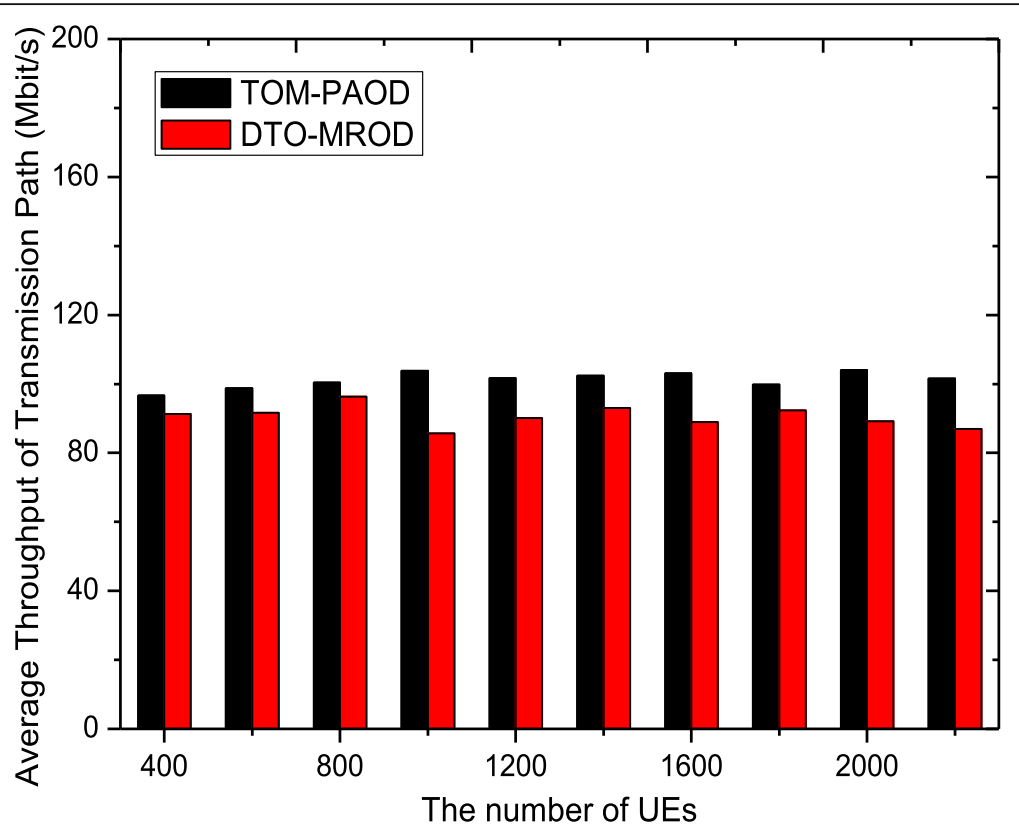

Fig. 3 Throughput performance comparison: variation trend of throughput in the two schemes with the number of UEs in the given region

communication complexity is $O(\max \{M, x \cdot N\})$. Then, it may receive $N$ request packets from $N$ UEs respectively and then send the corresponding response packets back, where its communication complexity is $O(N)$. Moreover, it must broadcast starting package for receiving mode selection (see line 4 in Algorithm 4) and then receive $N$ receiving mode selection information reporting packages from $N$ receiving UEs (see lines 6 10 in Algorithm 4), where its communication complexity is $\mathrm{O}(N)$.
Finally, it must broadcast starting package for power adjustment (see lines 14 and 24 in Algorithm 4) and then receive power adjustment feedback packages from $N$ receiving UEs (see lines 18 21 in Algorithm 4). Since the power adjustment game process are iterated $x$ times, the computational complexity is $O(x \cdot N)$. Based on the scenario described in the previous text, since $N$ is less than $M, \mathrm{O}(N)$ is better than $O(M)$. Therefore, the resulting communication complexity is $O(\max \{M, x \cdot N\})$.

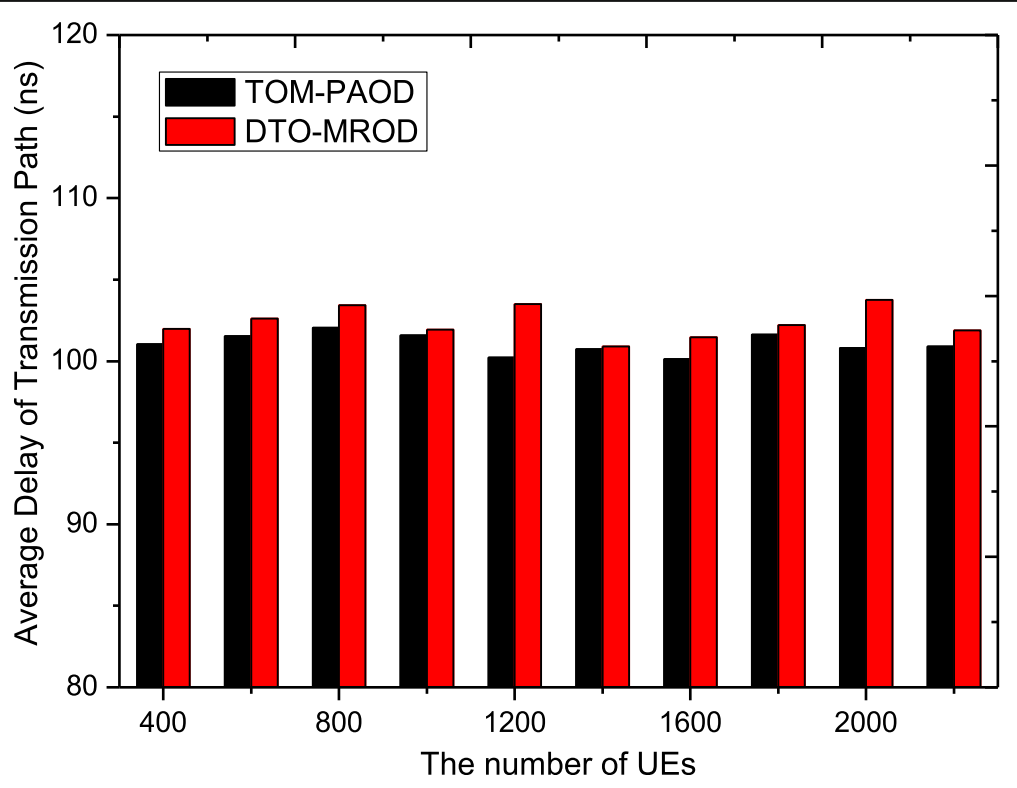

Fig. 4 Delay performance comparison: variation trend of delay in the two schemes with the number of UEs in the given region 


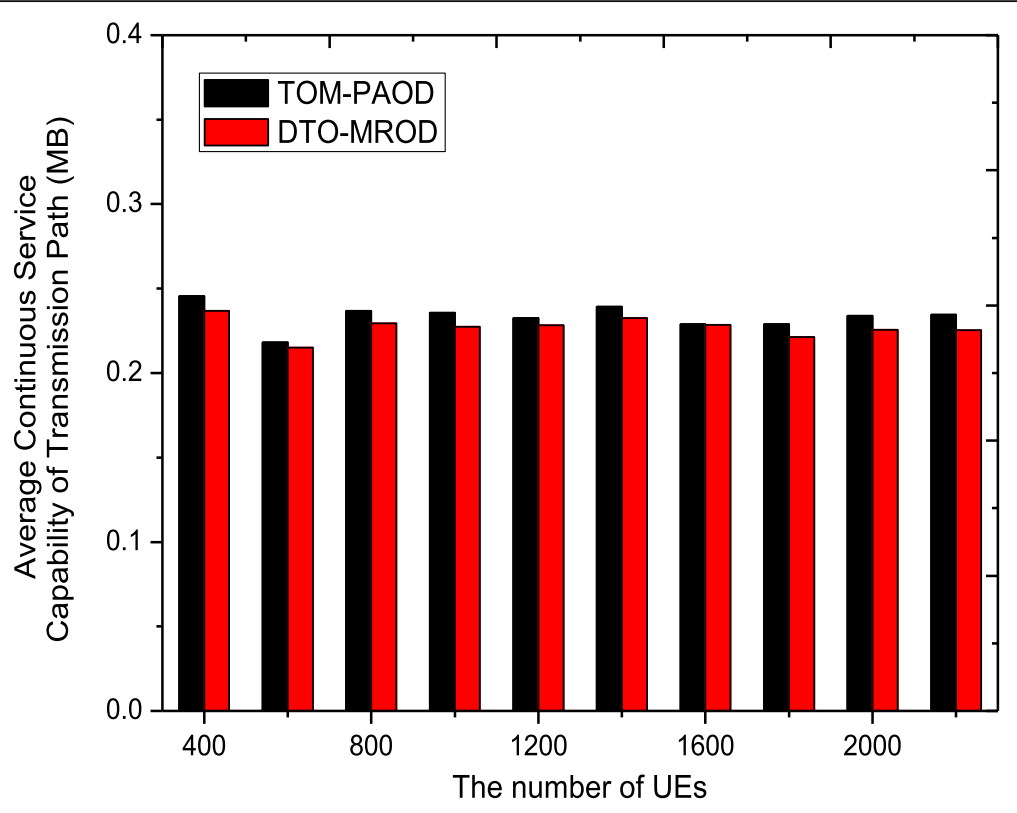

Fig. 5 Comparison of continuous service capacity: variation trend of continuous service capacity in the two schemes with the number of UEs in the given region

Based on the theoretical analysis for DTO-MROD, we know that the computational and communication complexities of DTO-MROD are $O\left(N \cdot \max \left\{\left|I_{i, \text { nei }}\right| \cdot\left|V_{j}\right|\right\}\right)$ and $O(M)$, respectively. Therefore, Table 1 lists a comparison of the communication and computational complexities between TOM-PAOD and DTO-MROD.

Table 1 shows that TOM-PAOD is better than DTO-MROD with respect to the computational complexity for the infrastructure node, where $O(\max \{|D|,|E|\}$-$\left.\max \left\{\left|I_{i, \text { nei }}\right| \cdot\left|V_{j}\right|\right\}\right)$ is better than $O\left(N \cdot \max \left\{\left|I_{i, \text { nei }}\right| \cdot\left|V_{j}\right|\right\}\right)$ since $\max \{|D|,|E|\}$ is less than $N$. Also, each receiving UE outperforms the infrastructure node in terms of computational complexity since $O\left(\max \left\{\left|V_{i}\right|, x, \max \left\{\left|V_{j}\right| \mid j \in V_{i}\right\}\right)\right.$ is better than $O\left(\max \{|D|,|E|\} \cdot \max \left\{\left|I_{i, \text { nei }}\right| \cdot\left|V_{j}\right|\right\}\right)$.

From Table 1, we also see that DTO-MROD may have an advantage on the communication complexity of the infrastructure node compared with TOM-PAOD. This is mainly because the communication complexity of game process used by the infrastructure node to assist each receiving UE for power adjustment may be greater than

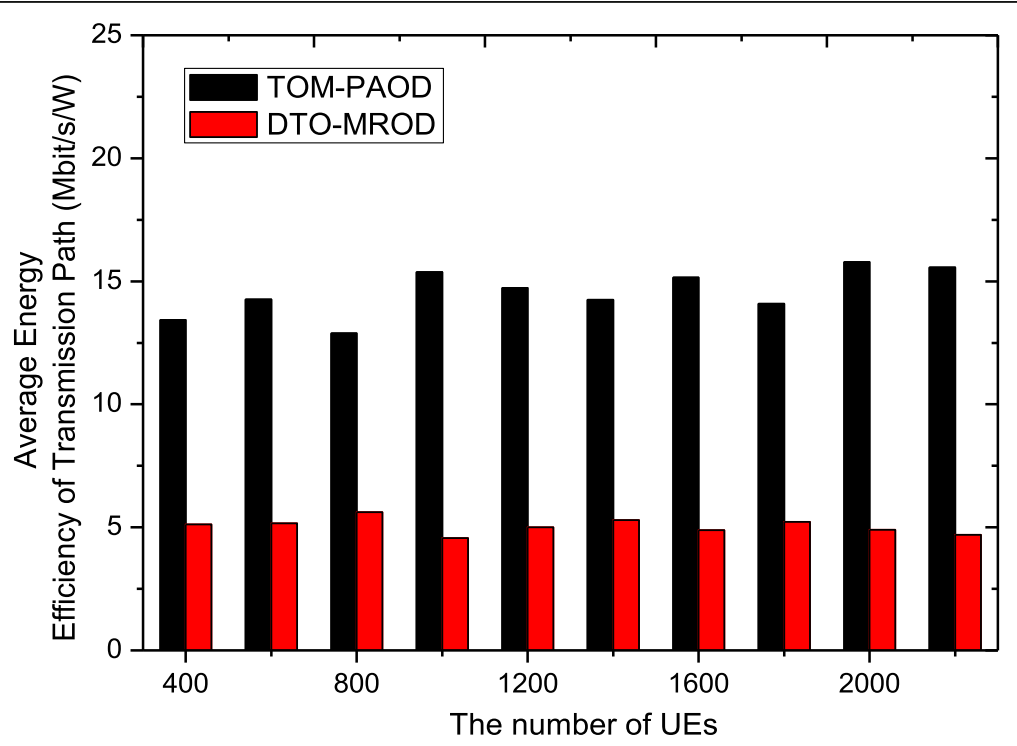

Fig. 6 Comparison of energy efficiency: variation trend of energy efficiency in the two schemes with the number of UEs in the given region 


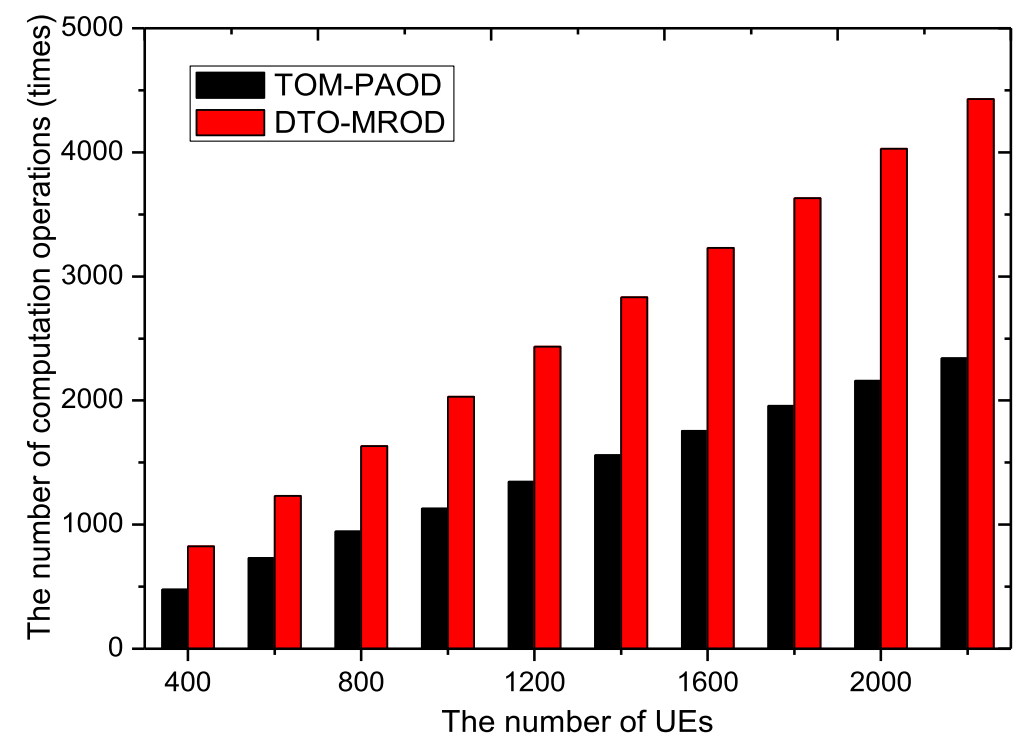

Fig. 7 Comparison of the number of computation operations: variation trend of the number of computation operations in the two schemes with the number of UEs in the given region

that of information collection. In addition, by designing game decision algorithm with faster convergence speed, we can control the communication complexity at the same order of magnitude as that of DTO-MROD.

\section{Performance evaluation}

\subsection{Simulation setting}

The work in this paper is the deepening research and further expansion of our previous works in $[15,47]$. The comparison of their characteristics is summarized in Table 2.

From Table 2, it is obvious that the most relevant scheme to our TOM-PAOD is the DTO-MROD in [15]. Therefore, the same simulation setting as that in [15] is adopted in this paper, where the parameter values used in our simulations have described in [15]. Please refer to the literature [15] for details.

However, TOM-PAOD differs from DTO-MROD in that we consider energy efficiency in terms of

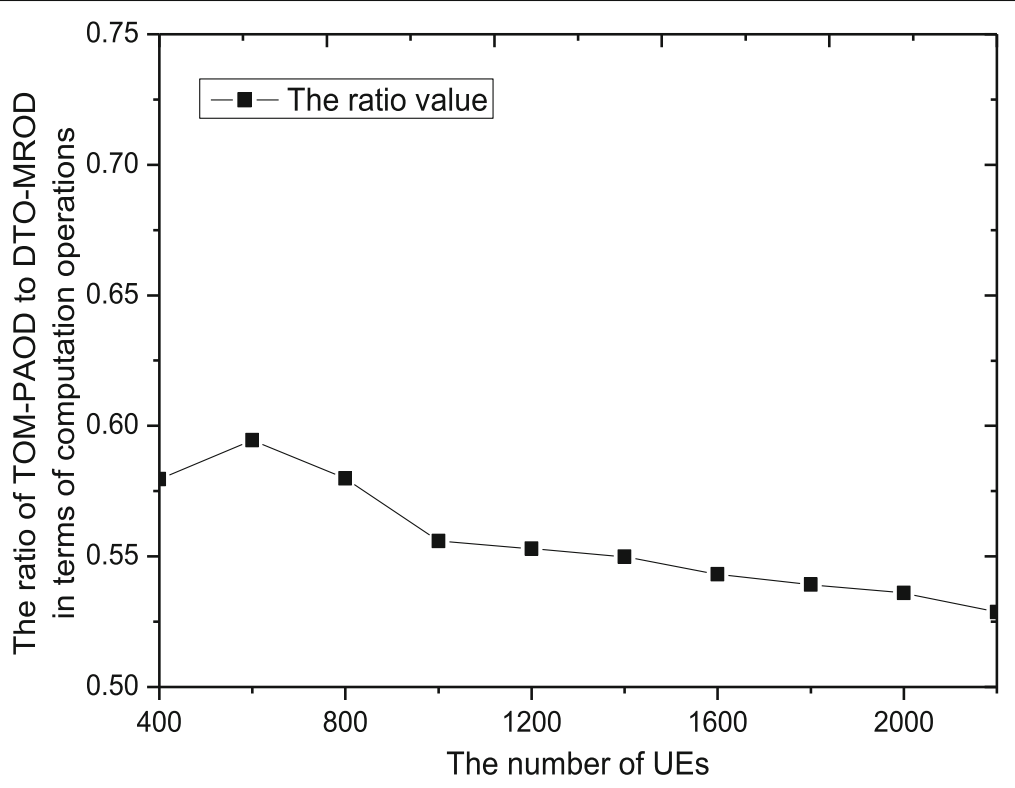

Fig. 8 Variation trend of the ratio with the number of UEs in the given region 


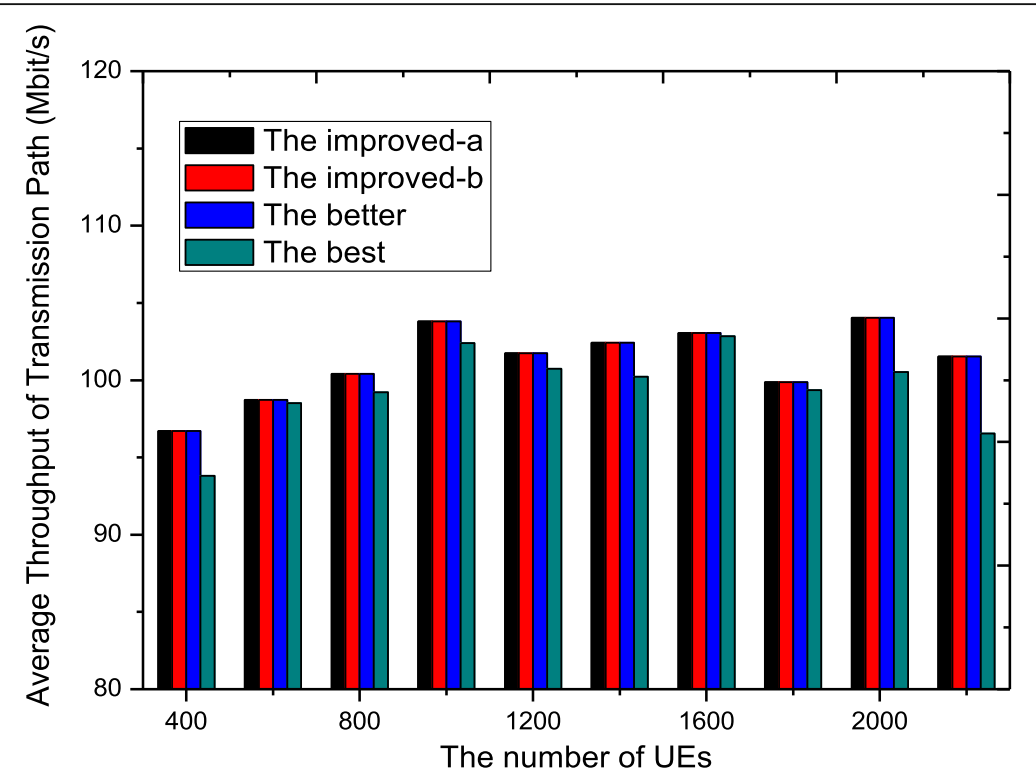

Fig. 9 Throughput performance comparison: variation trend of throughput of TOM-PAOD with the number of UEs in the given region, in which the different game decision algorithms are adopted respectively

throughput optimization and propose a network-assisted distributed mode for data receiving mode selection instead of a centralized mode from the same task.

In addition, there are the metrics for performance used in our simulations, including average downward transmission path throughput, average downward transmission path delay, average continuous service capacity for downward transmission path, average energy efficiency for downward transmission path, and average convergence time, which are described in detail in [47]. In order to save space, we do not repeat them.

\subsection{Simulation results and analysis}

Firstly, we estimate the performance of TOM-PAOD and DTO-MROD through the analysis of experimental results shown in Figs. 3, 4, 5, 6, 7, and 8. In TOM-PAOD, the improved-b proposed in this paper is adopted to adjust transmission power, and the step size for power control is set as $1 \mathrm{~mW}$.

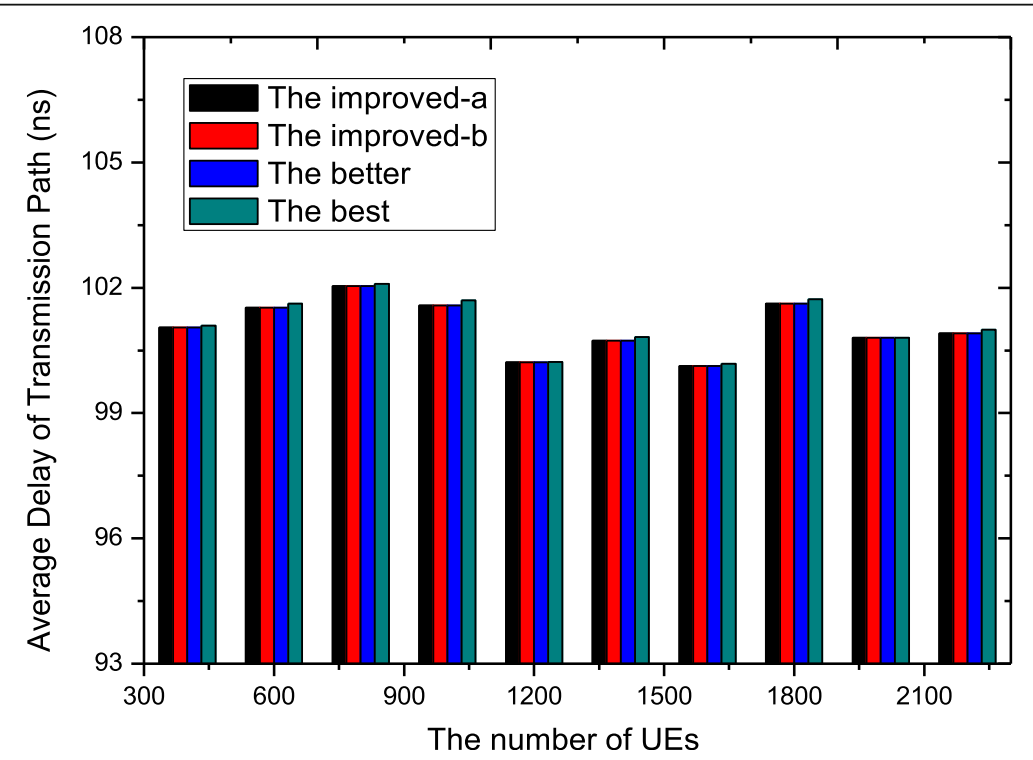

Fig. 10 Delay performance comparison: variation trend delay of TOM-PAOD with the number of UEs in the given region, in which the different game decision algorithms are adopted respectively 


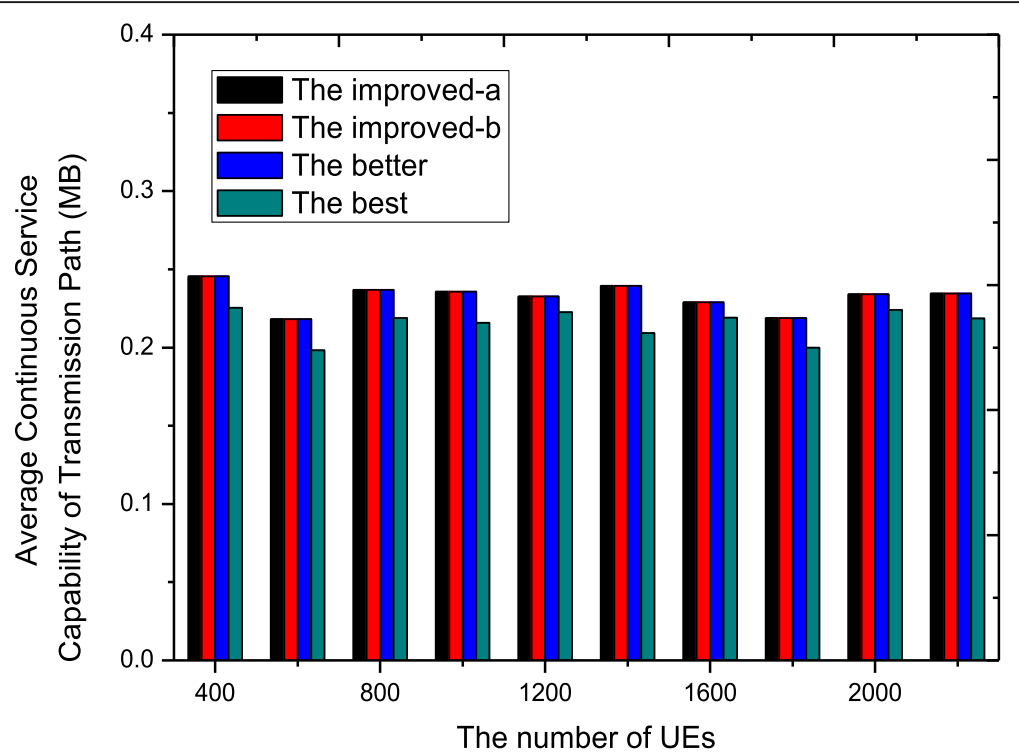

Fig. 11 Comparison of continuous service capacity: variation trend of continuous service capacity of TOM-PAOD with the number of UEs in the given region, in which the different game decision algorithms are adopted respectively

As shown in Fig. 3, the average downward transmission path throughput of TOM-PAOD is higher than that of DTO-MROD. The main reason is that, TOM-PAOD effectively reduces overlapping channel interference between outband D2D links through power adjustment, while the unified higher transmission power is employed in DTO-MROD, and thus, there is the greater overlapping channel interference between outband D2D links. Since average downward transmission path delay is generally inversely correlated with average downward transmission path throughput, the explanation of Fig. 3 can also apply to the scenario for delay variation shown in Fig. 4.

Figure 5 shows that TOM-PAOD outperforms DTO-MROD with respect to average continuous service capacity for downward transmission path. This is because, in TOM-PAOD, under the premise that the path throughput is basically not decreased, the powers of transmitting ends in a receiving path from the $e N B$ to a receiving UE is generally reduced through power

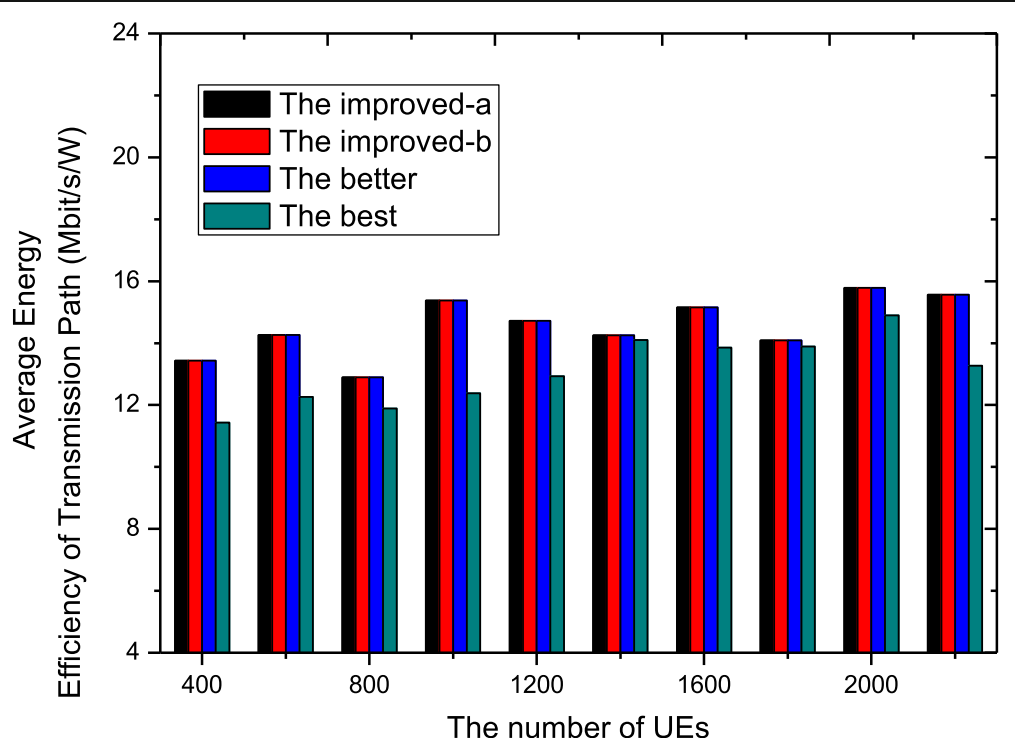

Fig. 12 Comparison of energy efficiency: variation trend of energy efficiency of TOM-PAOD with the number of UEs in the given region, in which the different game decision algorithms are adopted respectively 


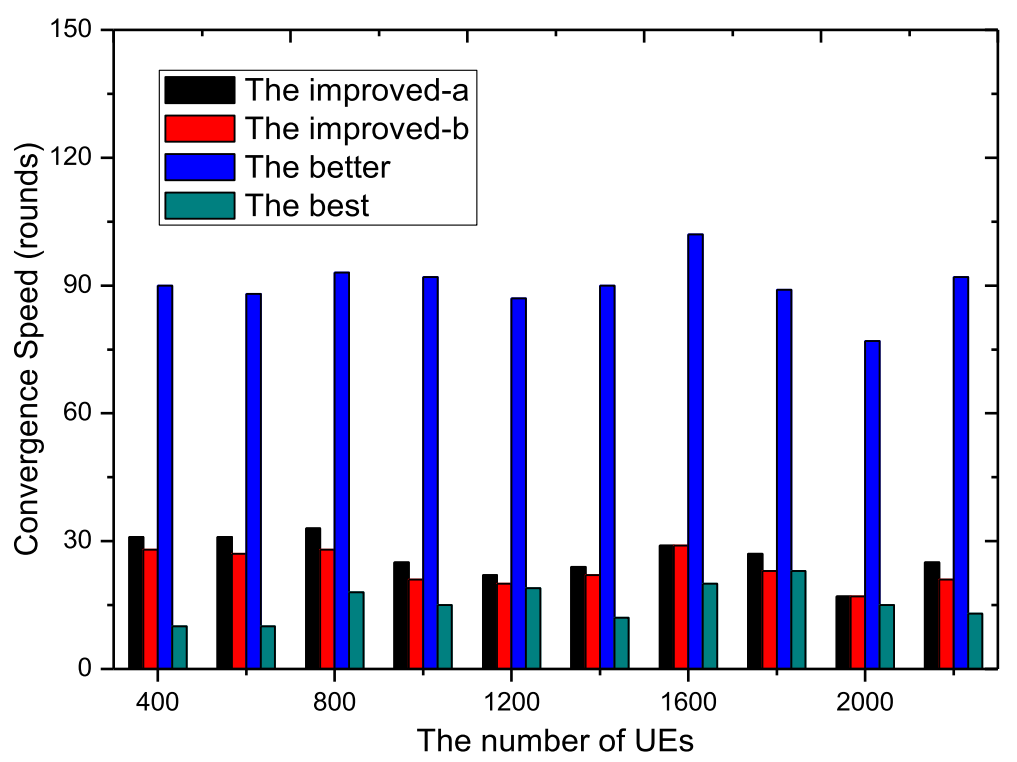

Fig. 13 Convergence performance comparison: variation trend of convergence time of TOM-PAOD with the number of UEs in the given region, in which the different game decision algorithms are adopted respectively

adjustment. Instead, DTO-MROD ignores power adjustment, which may cause unnecessary energy waste.

Therefore, the same amount of energy can be used to transmit more traffic if TOM-PAOD is used by an application system. This interpret also applies to the interpretation of phenomenon in Fig. 6. That is, it can be used to explain why the average energy efficiency of TOM-PAOD is higher than that of DTO-MROD.
From Fig. 7, we observe that, as the number of UEs increases, the number of computation operations executed by the infrastructure node (e.g., the $e N B$ or the ProSe Application Server) in DTO-MROD increases faster than that in TOM-PAOD. This phenomenon is mainly attributed to that, in DTO-MROD, the infrastructure node undertakes the computing task alone, and no any UE shares it, while each UE makes independent

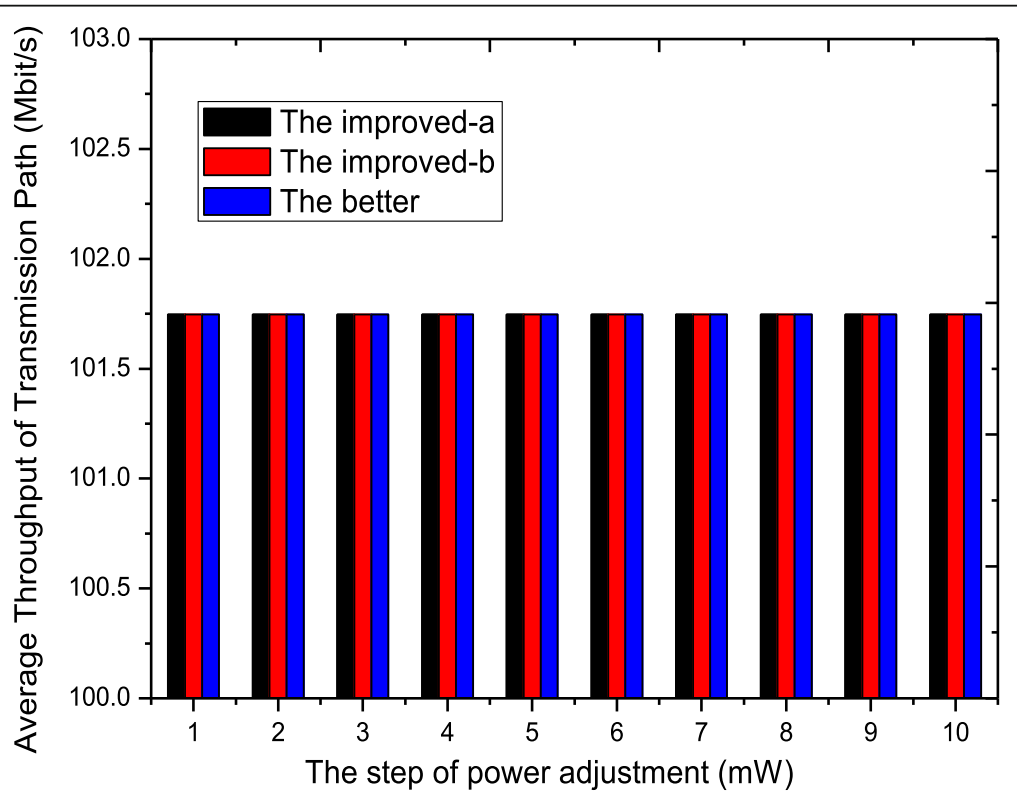

Fig. 14 Throughput performance comparison: variation trend of throughput of TOM-PAOD with the step size for power adjustment, in which the different game decision algorithms are adopted respectively 


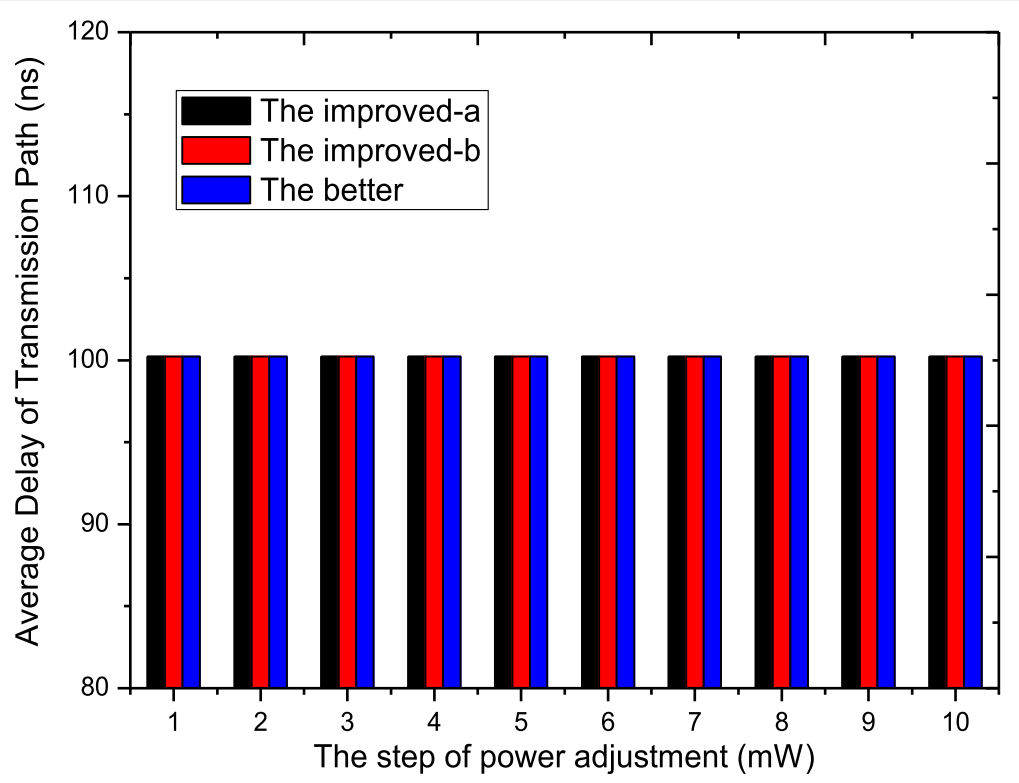

Fig. 15 Delay performance comparison: variation trend of delay of TOM-PAOD with the step size for power adjustment, in which the different game decision algorithms are adopted respectively

decisions with the aid of the infrastructure node in TOM-PAOD. Therefore, the communication load of the infrastructure node in TOM-PAOD can be reduced due to the sharing of individual UEs. This helps it adapt to network scale expansion, which is further confirmed by the result shown in Fig. 8.

Then, we evaluate the performance of our scheme when the four different game decision algorithms (i.e., the improved-a, the improved-b, the better response algorithm, and the best response algorithm) are employed in TOM-PAOD to adjust transmission power respectively.

Figures 9, 10, 11, 12, and 13 show that the performance change of TOM-PAOD with the number of UEs in the given region when the four different game decision algorithms are employed to adjust transmission power respectively, where the step size for power adjustment is set as $1 \mathrm{~mW}$ for the

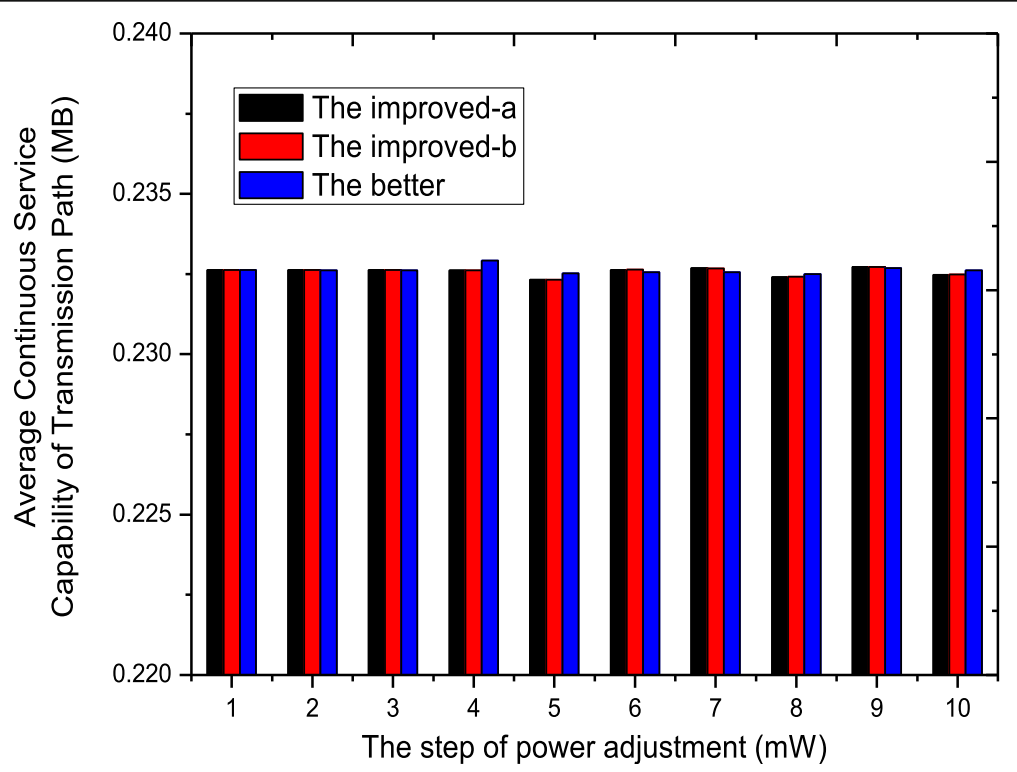

Fig. 16 Comparison of continuous service capacity: variation trend of continuous service capacity of TOM-PAOD with the step size for power adjustment, in which the different game decision algorithms are adopted respectively 


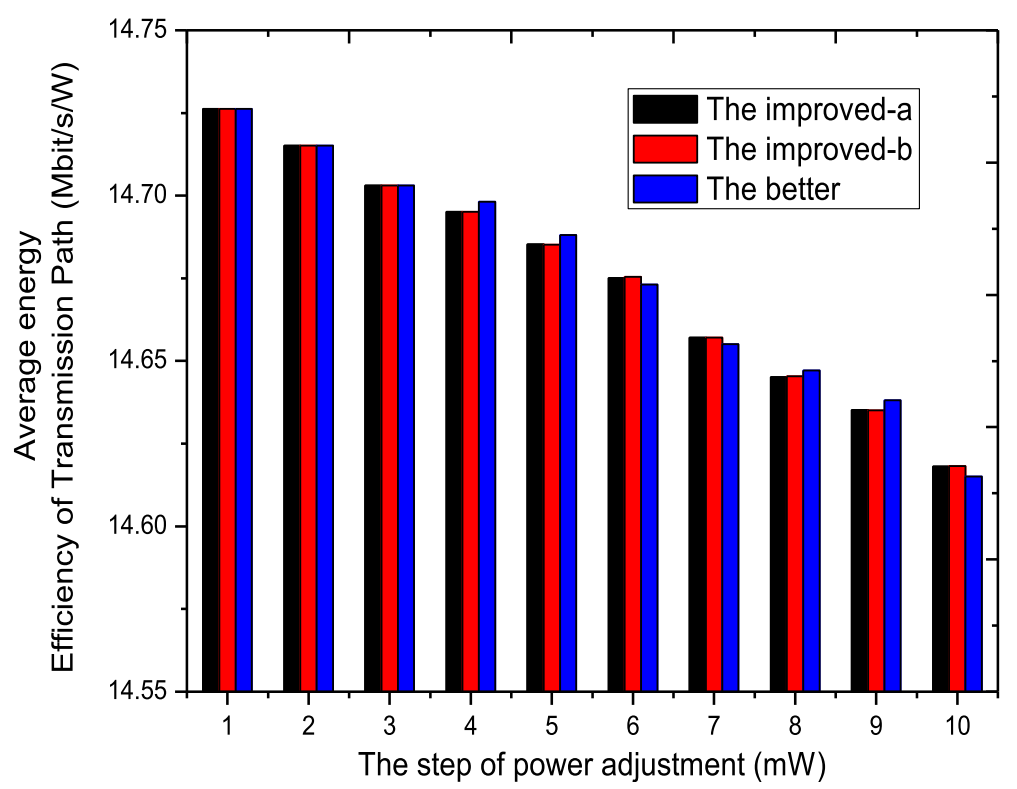

Fig. 17 Comparison of energy efficiency: variation trend of energy efficiency of TOM-PAOD with the step size for power adjustment, in which the different game decision algorithms are adopted respectively

improved-a, the improved-b, and the better response algorithm.

The TOM-PAOD scheme has a relatively poor performance when the best response algorithm is used to adjust transmission power. This is because the best response algorithm results in a biased steady-state for transmitting power distribution because of its greed
[52]. However, the best response algorithm has the fastest convergence speed.

On the other hand, although the TOM-PAOD scheme has a relatively good performance when the better response algorithm, the better response algorithm has the slowest convergence speed. The two improved response algorithms enhance the convergence speed of the better

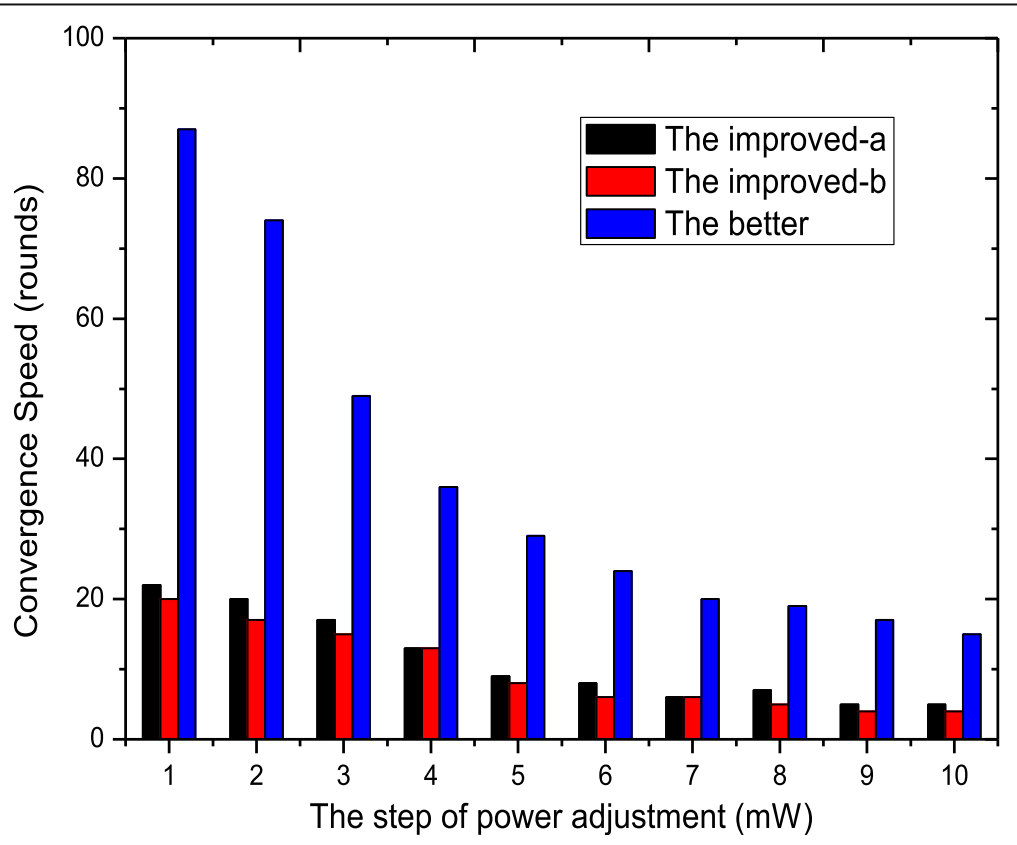

Fig. 18 Convergence performance comparison: variation trend of convergence time of TOM-PAOD with the step size for power adjustment, in which the different game decision algorithms are adopted respectively 
Table 3 Simulation parameters

\begin{tabular}{|c|c|c|}
\hline Description & Parameter & Value \\
\hline Transmitting antenna gain & $G_{t}$ & 1 \\
\hline Receiving antenna gain & $G_{r}$ & 1 \\
\hline Transmitting antenna height & $h_{t}$ & $1 \mathrm{~m}$ \\
\hline Receiving antenna height & $h_{r}$ & $1 \mathrm{~m}$ \\
\hline Transmitting power for UE & pue & $0.1 \mathrm{w}$ \\
\hline Transmitting power for eNB & $p_{c}$ & $10 w$ \\
\hline BER threshold for UE & $B E_{t h}$ & $10^{-10}$ \\
\hline Environment noise power & $N_{i}$ & $2 \times 10^{-11}$ \\
\hline Carrier signal wavelength & $\lambda$ & $0.1224 \mathrm{~m}$ \\
\hline System loss factor & L & 1 \\
\hline Crossover distance & $d_{\text {crossover }}$ & $103 \mathrm{~m}$ \\
\hline Path loss exponent & $a_{j i}$ & 2 or 4 \\
\hline Initial battery capacity & $e_{i, \mathrm{int}}$ & {$[0.05,0.2] J$} \\
\hline Transmitter electronics energy & $\varphi_{11}$ & $26.5 \mathrm{~nJ} / \mathrm{bit}$ \\
\hline Receiver electronics energy & $\varphi_{12}$ & $59.1 \mathrm{~nJ} / \mathrm{bit}$ \\
\hline Radio amplifier energy & $\varphi_{2}$ & $10 \mathrm{pJ} / \mathrm{bit} / \mathrm{m}^{2}$ or $0.0013 \mathrm{pJ} / \mathrm{bit} / \mathrm{m}^{4}$ \\
\hline Packet forwarding capacity & $t_{i}^{b}$ & 100 ns/bit \\
\hline Data packet length & M & 2500 bit \\
\hline
\end{tabular}

response algorithm, while they achieve the similar power adjustment performance to the better response algorithm.

Also, we see that the improved-b outperforms the improved-a in terms of convergence performance, which verifies the correctness of the method proposed in Subsection 3.3 by the simulation results. Although the best response algorithm has the fastest convergence speed among the four game decision algorithms, its worst performance restricts its practical application.

Figures 14, 15, 16, 17, and 18 show that the performance change of TOM-PAOD with the step size for power adjustment when the different game decision algorithms are employed to adjust transmission power respectively, where the number of UEs in the given region is set as 1200. Since the best response algorithm has nothing to do with step size, it is excluded from Figs. 14, 15, 16, 17, and 18 .

Differing from the case that throughput, delay, and continuous service capacity show a random fluctuation characteristic as node density changes, these metrics are hardly affected by the variation of step size for power adjustment. However, from careful observation, we can see that the performance is very slightly reduced with the increase of step size. This is because the larger step size is more difficult to match the exact convergence point. Although this mismatch has minimal impact on throughput, delay, and continuous service capacity, its effect on energy efficiency is more obvious. As shown in Fig. 17, energy efficiency declines as the step size grows.
From Fig. 18, we see that the larger step size results in the smaller difference of convergence speed between the better response algorithm and the two improved response algorithms. This is because the two improved response algorithms only adopt a large step size in the first round, and thus, the speed advantage of the first round will be undermined as step size increases.

\section{Conclusions}

In this paper, we explore throughput optimization problem by applying a multi-hop relay-aided outband D2D communication to future cellular networks and employing a potential game-based tool for designing power adjustment method and propose a network-assisted distributed processing architecture for solving the above optimization problem, which consists of the three cascaded stages related to receiving mode selection, verification for relay selection, and transmission power adjustment. In receiving mode selection stage, with the aid of the $e N B$, each receiving UE independently determines its data receiving mode. In verification for relay selection stage, the $e N B$ is responsible for verifying whether each selected relay can improve throughput for the corresponding receiving UE in a centralized manner. In transmission power adjustment, under the unified coordination of the $e N B$, each receiving UE independently adjusts the powers of the transmitting ends on its data receiving path.

Although the proposed scheme has better performance in terms of throughput, delay, energy efficiency, 
and continuous service ability when compared with the existing typical work, it is assumed that each idle UE with no interest in receiving from the $e N B$ is willing to provide a relaying service as the premise. In fact, any UE is usually rational, selfish, or even malicious and may be reluctant to act as a relay without any reward. Therefore, we plan to explore incentive mechanisms to encourage idle UEs to participate in outband D2D relaying services. In addition, the research combining incentive mechanism with the social awareness method [53] and the trust mechanism for throughput improvement is also an interesting future direction.

\section{Methods/experimental}

The same simulation setting as that in [15] is adopted in this paper, where the parameter values used in our simulations have described in [15]. For the convenience of readers, we also list them as follows.

The simulation network is a circular region with a radius of $500 \mathrm{~m}$, and the $e N B$ is located in the center of the region. In addition, the parameter values employed in our simulation are given in Table 3.

\begin{abstract}
Abbreviations
5G: The fifth generation cellular network; BER: Bit error rate; BS: Base station; CAPEX: Capital expenditure; CSI: Channel state information; D2D: Device-todevice communication; DORE: The D2D opportunistic relay with QoS enforcement scheme; DTO-MROD: The method for downlink throughput optimization by multi-hop relay-assisted outband D2D communications; eNB: Evolved node B; IoT: Internet of Things; NE: Nash Equilibria; OPEX: Operating expenditure; OPF: Ordinal potential function; OPG: Ordinal potential game; ProSe: Proximity-based service; QoS: Quality of service; RB: Resource block; RMS: Receiving mode selection; SINR: Signal-tointerference-plus-noise ratio; TOM-PAOD: The throughput optimization method based on power adjustment and outband D2D communication; TPA: Transmission power adjustment; UE: User equipment; VRS: Verification for relay selection
\end{abstract}

\section{Acknowledgements}

The authors would like to acknowledge the anonymous reviewers for their thoughtful comments.

\section{Funding}

This work was supported in part by the National Natural Science Foundation of China $(61873352,61272494)$

\section{Availability of data and materials}

Not applicable

\section{Authors' contributions}

JSG presented the scheme and designed the experiments. KZ did the experiments, analyzed the data, and explained the simulation results. JSG drafted this paper, and all authors read and approved it.

\section{Authors' information}

Jinsong Gui received the PhD from Central South University, China, in 2008. He is currently a Professor in Department of Computer Science and Technology, School of Information Science and Engineering, Central South University, China. Kai Zhou is currently a Master Student working in Department of Computer Science and Technology, School of Information Science and Engineering, Central South University, China.

\section{Competing interests}

The authors declare that they have no competing interests.

\section{Publisher's Note}

Springer Nature remains neutral with regard to jurisdictional claims in published maps and institutional affiliations.

Received: 1 May 2018 Accepted: 11 October 2018

Published online: 25 October 2018

\section{References}

1. Cisco visual networking index: forecast and methodology, 2016-2021 (CISCO White Paper, 2017). https://www.cisco.com/c/en/us/solutions/collateral/ service-provider/visual-networking-index-vni/complete-white-paper-c11481360.html

2. J.L. Gao, J.X. Wang, P. Zhong, H.D. Wang, On threshold free error detection for industrial wireless sensor networks. IEEE Trans. Ind. Inf.. https://doi.org/10. 1109/ TII.2017.2785395

3. Q. Liu, A.F. Liu, On the hybrid using of unicast-broadcast in wireless sensor networks. Comput. Electr. Eng. (2017). https://doi.org/10.1016/j. compeleceng.2017.03.004

4. A.F. Liu, S. Zhao, High performance target tracking scheme with low prediction precision requirement in WSNs. Int. J. Ad Hoc Ubiquitous Comput. (2017) http://www.inderscience.com/info/ingeneral/forthcoming. php?jcode=ijahuc

5. J. Jiang, G. Han, L. Shu, S. Chan, K. Wang, A trust model based on cloud theory in underwater acoustic sensor networks. IEEE Trans. Ind. Inf. 13, 342350 (2017)

6. M. Mukherjee, L. Shu, H. L, et al., Sleep scheduling in industrial wireless sensor networks for toxic gas monitoring. IEEE Wirel. Commun. 24, 106-112 (2017)

7. Y.L. Xiao, S.G. Zhang, J.X. Wang, C.Z. Zhu, A novel indoor localization algorithm for efficient mobility management in wireless networks. Wirel. Commun. Mob. Comput. 9517942, 1-9517942 (2018)

8. R.T. Hermeto, A. Gallais, F. Theoleyre, Scheduling for IEEE802.15.4-TSCH and slow channel hopping MAC in low power industrial wireless networks: a survey. Comput. Commun. 114, 84-105 (2017)

9. X. Liu, Y. Liu, N.X. Xiong, N. Zhang, A.F. Liu, H. Shen, C. Huang, Construction of large-scale low cost deliver infrastructure using vehicular networks. IEEE Access 6(1), 21482-21497 (2018)

10. X. Liu, M.X. Dong, K. Ota, L. Yang, A.F. Liu, Trace malicious source to guarantee cyber security for mass monitor critical infrastructure. J. Comput. Syst. Sci. (2016). https://doi.org/10.1016/j.jcss.2016.09.008

11. T. Li, N.X. Xiong, J. Gao, H. Song, A.F. Liu, Z.W. EZeng, Reliable code disseminations through opportunistic communication in vehicular wireless networks. IEEE Access (2018). https://doi.org/10.1109/ACCESS.2018.2870928

12. I.F. Akyildiz, S. Nie, S.C. Lin, M. Chandrasekaran, 5G roadmap: 10 key enabling technologies. Comput. Netw. 106, 17-48 (2016)

13. A. Asadi, V. Mancuso, R. Gupta, in Proceedings of the IEEE INFOCOM Conference. An SDR-based experimental study of outband D2D communications (2016), pp. 1-9

14. A. Asadi, V. Mancuso, R. Gupta, DORE: an experimental framework to enable outband D2D relay in cellular networks. IEEE/ACM Trans. Networking 25, 2930-2943 (2017)

15. K. Zhou, J.S. Gui, N.X. Xiong, Improving cellular downlink throughput by multi-hop relay-assisted outband D2D communications. EURASIP J. Wirel. Commun. Netw. 209 (2017). https://doi.org/10.1186/s13638-017-0998-9

16. A.I. Asad, A.Q. Wang, V. Mancuso, A survey on device-to-device communication in cellular networks. IEEE Commun. Surveys Tuts. 16, 18011819 (2014)

17. P. Mach, Z. Becvar, T. Vanek, In-band device-to-device communication in OFDMA cellular networks: a survey and challenges. J. Comput. Syst. Sci. 17, 1885-1922 (2015)

18. J.J. Liu, N. Kato, J.F. Ma, N. Kadowaki, Device-to-device communication in LTE-advanced networks: a survey. IEEE Commun. Surv. Tutorials 17, 19231940 (2017)

19. S.N. Swain, R. Thakur, C.S.R. Murthy, Design and stochastic geometric analysis of an efficient Q-learning based physical resource block allocation scheme to maximize the spectral efficiency of device-to-device overlaid cellular networks. Comput. Netw. 119(4), 71-85 (2017)

20. Q.Q. Wu, G.Y. Li, W. Chen, Energy-efficient D2D overlaying communications with spectrum-power trading. IEEE Trans. Wirel. Commun. 16(7), 4404-4419 (2017) 
21. J.H. Dai, J.J. Liu, Y.P. Shi, Analytical modeling of resource allocation in D2D overlaying multihop multichannel uplink cellular networks. IEEE Trans. Veh. Technol. 66(8), 6633-6644 (2017)

22. Z.Y. Yang, Y.W. Kuo, Efficient resource allocation algorithm for overlay D2D communication. Comput. Netw. 124, 61-71 (2017)

23. Z.J. Lin, L.F. Huang, Y.J. Li, Analysis of transmission capacity for multi-mode D2D communication in mobile networks. Pervasive Mob. Comput. 41, 179$191(2017)$

24. X.L. Liu, N. Ansari, Green relay assisted D2D communications with dual batteries in heterogeneous cellular networks for loT. IEEE Internet Things J. 4(5), 1707-1715 (2017)

25. D.H. Kim, S.J. Oh, J.T. Lim, Multi-channel-beased scheduling for overlay inband device-to-device communications. Wirel. Netw 23(8), 2587-2600 (2017)

26. A. Pyattaev, K. Johnsson, S. Andreev, Y. Koucheryavy, in Proceedings of IEEE Wireless Communications and Networking Conference Workshops (WCNCW), Shanghai, China. 3GPP LTE traffic offloading onto WiFi direct (2013), pp. 135-140

27. A. Pyattaev, K. Johnsson, A. Surak, R. Florea, S. Andreev, Y. Koucheryavy, in Proceedings of IEEE Wireless Communications and Networking Conference (WCNC), Istanbul, Turkey. Network-assisted D2D communications: implementing a technology prototype for cellular traffic offloading (2014), pp. 3266-3271

28. L. Militano, M. Condoluci, G. Araniti, A. Molinaro, A. lera, F. Fitzek, in Proceedings of IEEE International Conference on Communications (ICC), Sydney, Australia. Wi-fi cooperation or D2D-based multicast content distribution in LTE-A: a comparative analysis (2014), pp. 296-301

29. S. Andreev, A. Pyattaev, K. Johnsson, O. Galinina, Y. Koucheryavy, Cellular traffic offloading onto network-assisted device-to-device connections. IEEE Commun. Mag. 52, 20-31 (2014)

30. S. Andreev, O. Galinina, A. Pyattaev, K. Johnsson, Y. Koucheryavy, Analyzing assisted offloading of cellular user sessions onto D2D links in unlicensed bands. IEEE J. Sel. Areas Commun. 33, 67-80 (2015)

31. A. Hematian, W. Yu, C. Lu, D. Griffith, N. Golmie, in Proceedings of conference on research in adaptive and convergent systems (RACS), Univ so Denmark, Odense, Denmark. A clustering-based device-to-device communication to support diverse applications (2016), pp. 97-102

32. A. Hematian, W. Yu, C. Lu, D. Griffith, N. Golmie, Towards clustering-based device-to-device communications for supporting applications. Appl. Comput. Rev. 17, 35-48 (2017)

33. A. Asadi, V. Mancuso, Network-assisted outband D2D-clustering in $5 G$ cellular networks: theory and practice. IEEE Trans. Mob. Comput. 16, 2246-2259 (2017)

34. H.L. Zhang, Y. Liao, L.Y. Song, D2D-U: device-to-device communications in unlicensed bands for 5 G system. IEEE Trans. Wirel. Commun. 16, 3507-3519 (2017)

35. C. Karakus, S. Diggavi, Enhancing multiuser MIMO through opportunistic D2D cooperation. IEEE Trans. Wirel. Commun. 16, 5616-5629 (2017)

36. J.S. Gui, J. Deng, A topology control approach reducing construction cost for lossy wireless sensor networks. Wirel. Pers. Commun. 95(3), 2173-2202 (2017)

37. Z.Y. Zhou, M.X. Dong, Z. Chang, B. Gu, in Proceedings of IEEE ICCC'15, Shenzhen, China. Combined centralized and distributed resource allocation for green D2D communications (2015), pp. 1-6

38. Z.Y. Zhou, M.X. Dong, K. Ota, G.J. Wang, L.T. Yang, Energy-efficient resource allocation for D2D communications underlaying cloud-RAN-based LTE-A networks. IEEE Internet Things 3(3), 428-438 (2016)

39. Z.Y. Zhou, G.F. Ma, C. Xu, Z. Chang, T. Ristaniemi, in Proceedings of IEEE ICC'16, Kuala Lumpur, Malaysia. Energy-efficient resource allocation in cognitive D2D communications: a game-theoretical and matching approach (2016), pp. 1-6

40. D. Monderer, L. Shapley, Potential games. Games Econom. Behav. 14, 124 143 (1996)

41. S. Buzzi, G. Colavolpe, D. Saturnino, A. Zappone, Potential games for energyefficient power control and subcarrier allocation in uplink multicell OFDMA systems. IEEE J. Sel. Topics Signal Process. 6(2), 89-103 (2012)

42. Q.D. La, Y.H. Chew, B.H. Soong, Performance analysis of downlink multi-cell OFDMA systems based on potential game. IEEE Trans. Wirel. Commun. 11(9), 3358-3367 (2012)

43. A. Abrardo, M. Moretti, Distributed power allocation for D2D communications underlaying/overlaying OFDMA cellular networks. IEEE Trans. Wirel. Commun. 16, 1466-1479 (2017)

44. 3rd Generation Partnership Project, Technical Specification Group Services and System Aspects, Study on Architecture Enhancements to Support Proximity-Based Services (ProSe) (Release 12), document TR 23.703 V12.0.0 (3rd
Generation Partnership Project, 2014). https://www.arib.or.jp/english/html/ overview/doc/STD-T63v11 00/5_Appendix/Rel12/23/23703-c00.pdf

45. 3rd Generation Partnership Project, Technical Specification Group Services and System Aspects, Proximity-Based Services (ProSe); Stage 2 (Release 13), Document TR 23.303 V13.0.0 (3rd Generation Partnership Project, 2015) https://www.etsi.org/deliver/etsi_ts/123400_123499/123468/13.03.00_60/ts_ 123468v130300p.pdf

46. 3rd Generation Partnership Project, Technical Specification Group RAN, Study on LTE Device to Device Proximity Services (ProSe) Radio Aspects (Release 13), Document TR 36.843 V12.0.1 (3rd Generation Partnership Project, 2015). https://www.arib.or.jp/english/html/overview/doc/STD-T63v11_00/5_ Appendix/Rel12/23/23703-c00.pdf

47. J.S. Gui, J. Deng, Multi-hop relay-aided underlay D2D communications for improving cellular coverage quality. IEEE Access 6(1), 14318-14338 (2018). https://doi.org/10.1109/ACCESS.2018.2796247.

48. J.S. Gui, Y.J. Lu, X.H. Deng, A.F. Liu, Flexible resource allocation adaptive to communication strategy selection for cellular clients using Stackelberg game. Ad Hoc Netw. 66, 64-84 (2017)

49. J.S. Gui, L.H. Hui, N.X. Xiong, Enhancing cellular coverage quality by virtual access point and wireless power transfer. Wirel. Commun. Mob. Comput., 9218239 (2018). https://doi.org/10.1155/2018/9218239

50. S. Maghsudi, S. Stanczak, Hybrid centralized-distributed resource allocation for device-to-device communication underlaying cellular networks. IEEE Trans. Veh. Technol. 65, 2481-2495 (2016)

51. G. Katsinis, E.E. Tsiropoulou, S. Papavassiliou, Joint resource block and power allocation for interference management in device to device underlay cellular networks: a game theoretic approach. Mobile Netw. Appl. 22, 539551 (2017)

52. R.S. Komali, A.B. MacKenzie, R.P. Gilles, Effect of selfish node behavior on efficient topology design. IEEE Trans. Mob. Comput. 7, 1057-1070 (2008)

53. J.L. Gao, J.X. Wang, J.B. He, F.X. Yan, Against signed graph deanonymization attacks on social networks. Int. J. Parallel Prog. (2017). https://doi.org/10. 1007/s10766-017-0546-6

\section{Submit your manuscript to a SpringerOpen ${ }^{\circ}$ journal and benefit from:}

- Convenient online submission

Rigorous peer review

- Open access: articles freely available online

High visibility within the field

- Retaining the copyright to your article

Submit your next manuscript at $>$ springeropen.com 